\title{
Evaluer les réformes des exonérations générales de cotisations sociales
}

Mathieu Bunel, Celine EMONd, YANNICK L’HorTy

TEPP - Travail, Emploi et Politiques Publiques - FR CNRS 3126 


\title{
Evaluer les réformes des exonérations générales de cotisations sociales
}

\author{
Mathieu BUNEL ${ }^{*}$, Céline EMOND ${ }^{* *}$, Yannick L'HORTY***
}

\begin{abstract}
Juillet 2012
Résumé ${ }^{1}$

Les exonérations générales de cotisations sociales employeurs, dont le budget annuel dépasse 20 milliards d'euros, exercent des effets différenciés selon les secteurs d'activité. Nous évaluons ces effets sur le coût du travail puis sur l'emploi en partant de distributions de salaires tirées des Déclarations annuelles de données sociales (DADS), recalées avec les données de l'Agence centrale des organismes de sécurité sociale (Acoss) et d'élasticités de la demande de travail à son coût estimées sur des données d'entreprises.

D'après nos simulations, obtenues à l'aide d'un outil ad hoc d'évaluation ex ante, baptisé SISMICs, qui est un Simulateur Inter-Sectoriel pour la Mesure des Impacts des Cotisations Sociales, la suppression de l'ensemble des exonérations générales de cotisations sociales conduirait à une hausse du coût du travail de l'ordre de 4,4\% pour l'ensemble des salariés et de 12,3\% pour les salariés qui bénéficient des exonérations. Les destructions d'emplois associées à ce renchérissement $d u$ coût du travail seraient comprises entre 500000 et 610000 emplois dont près de la moitié dans les secteurs intenses en main-d'œuvre alors qu'ils ne représentent que 30\% de l'ensemble de l'emploi des secteurs privés. Les destructions d'emplois directs se concentreraient principalement sur les bas salaires avec $85 \%$ des emplois perdus qui seraient compris entre le Smic et 1,3 fois le Smic.

Nous évaluons également les effets ex ante de plusieurs scenarii de réformes qui modifient un ou plusieurs paramètres du barème des exonérations (sa pente, son seuil d'extinction, son montant maximal, ou son système de décote avantageant les entreprises de moins de 20 salariés). A enveloppe budgétaire donnée, ces réformes sont plus ou moins ciblées sur les bas salaires et affectent de manière hétérogène les entreprises des secteurs intenses en main-d'œuvre. Selon les scenarii, l'effet de ces réformes sur l'emploi varie du simple au double. Nos résultats indiquent que les réformes les moins défavorables à l'emploi, sont celles qui préservent le plus les bas salaires et les entreprises qui les emploient.
\end{abstract}

Code JEL: C14, J3, J38

Mots-clés : évaluation des politiques publiques, exonérations de cotisations sociales, coût du travail

\footnotetext{
* Mathieu BUNEL Université de Caen, CREM et TEPP (FR CNRS n³126), 19 rue Claude Bloch, 14000 Caen,. matthieu.bunel@unicaen.fr

Céline EMOND, Université Paris-Est, ERUDITE et TEPP (FR CNRS n³126), 5 boulevard Descartes, Champs sur Marne 77454 Marne la Vallée cedex 2, celine.emond@univ-mlv.fr

**** Yannick L'HORTY, Université Paris-Est, ERUDITE et TEPP (FR CNRS n ${ }^{\circ}$ 126), 5 boulevard Descartes, Champs sur Marne 77454 Marne la Vallée cedex 2, Yannick.lhorty@univ-mlv.fr

${ }^{1}$ Cette étude a bénéficié de l'appui des fédérations professionnelles des secteurs à forte intensité de main d'œuvre opérationnelle. En tant que financeurs, Fédération des Entreprises de Propreté et Services Associés (FEP) ; Comité de Liaison des Industries de Main d'œuvre (CLIMO) ; Union des Entreprises de la Sécurité Privée (USP) ; Professionnels de l'intérim, services et métiers de l'emploi (PRISME); Syndicat des Opérateurs Postaux (SOP) ; Syndicat de la Distribution Directe (SDD); Syndicat des Auxiliaires de la Manutention et de l'Entretien pour le Rail et l'Air (SAMERA). En tant que soutien, Union des Métiers des Industries de l'Hôtellerie (UMIH) ; Groupement des Professions de Services (GPS) ; Fédération du Service aux Particuliers (FESP) ; Fédération des Entreprises du Commerce et de la Distribution (FCD); Syndicat National de la Restauration Collective (SNRC) ; Fédération Française du Bâtiment (FFB) ; Syndicat National de l'Alimentation et de la Restauration Rapide (SNARR) ; Fédération Nationale des Activités de la Dépollution et de l'Environnement (FNADE) ; Chambre Syndicale de l'Assistance en Escale (CSAE). Cependant, ce document n'engage que ses auteurs et ne représente pas la position de ces fédérations.

Cette étude a été présentée au colloque «Trajectoires, Emplois et Politiques publiques » (Caen, juin 2012) et au 61 ème congrès de l'AFSE (Paris, juillet 2012).
} 


\section{INTRODUCTION}

Les différents dispositifs d'allègements généraux de cotisations sociales qui ont été progressivement déployés en France depuis le début des années quatre-vingt-dix, sont toujours restés ciblés sur les bas et les moyens salaires. Depuis la réforme Fillon de 2003, ces dispositifs représentent un budget annuel de plus de 20 milliards d'euros et prennent la forme d'une exonération dégressive, de 26 points de cotisations employeurs au niveau du SMIC ( $28,1 \%$ pour les entreprises de moins de 20 salariés) et qui diminue régulièrement avec le salaire jusqu'à s'annuler à 1,6 Smic. Pour une entreprise donnée, le montant des exonérations dépend donc fortement de la distribution des salaires, qui renvoie pour l'essentiel à la structure de ses qualifications. C'est la raison pour laquelle certains secteurs d'activité riches en main-d'œuvre vont être très concernés par ces dispositifs, tandis que pour d'autres secteurs plus intenses en travail qualifié et en capital, ces dispositifs n'auront pas ou peu d'effets sur le montant des prélèvements sociaux à la charge des employeurs.

Un petit nombre de secteurs d'activité, essentiellement issus des services, sont donc très concernés par ces allègements de cotisations sociales ${ }^{2}$. Pour autant, depuis vingt ans, les travaux d'évaluation n'ont pas véritablement fait jouer un rôle actif à cette dimension. Les évaluations ex ante ont mobilisé pour l'essentiel des modélisations agrégées où l'économie n'est pas composée de plusieurs secteurs d'activité et où la main-d'œuvre elle-même n'est composée que de deux ou trois catégories de main-d'œuvre (Laffargue [1996] ; Malinvaud [1998] ; Salanié, [1999]; Audric et alii [2000]; Laffargue [2000]; Laroque et Salanié [2000] ; Cahuc [2003], Doisy et alii [2004] ; Chéron et alii [2005]; Langot [2011]). Il est d'ailleurs frappant de constater qu'une synthèse récente des travaux d'évaluation réalisés de 1993 à 2009 n'accorde aucune place à la dimension sectorielle du dispositif (Ourliac et Nouveau, [2012]).

Les évaluations ex post et les études qui mobilisent des données individuelles d'entreprises, n'ont quant à elles que rarement donné un rôle actif aux effets sectoriels de ces dispositifs. L'évaluation de Crépon et Desplatz [2001] distingue uniquement l'industrie et le tertiaire. Le travail de Gafsi et alii [2004] décompose l'évolution de l'emploi et du coût du travail à un niveau sectoriel intermédiaire, en 16 secteurs, mais la problématique est de rechercher des invariants entre ces secteurs afin d'estimer plusieurs paramètres d'intérêt à un niveau agrégé. L'étude de Jamet [2005] part également du constat que les évolutions de l'emploi et du coût du travail diffèrent parfois fortement selon les secteurs d'activité et en tire une mesure du biais d'agrégation entre les élasticités sectorielles et macroéconomiques, mais elle conclut que ce biais est limité et que les effets d'entraînement entre secteurs sont compensés parles effets de concurrence, justifiant finalement les approches agrégées qui négligent ces biais en utilisant des élasticités microéconomiques dans des maquettes macroéconomiques. Une exploration purement théorique de Malinvaud [2002] sur l'agrégation des demandes de travail non qualifié souligne néanmoins que «le biais induit par un raisonnement intégralement agrégé sera proportionnel à un indicateur d'hétérogénéité3 $»$ et qu'une «approche

\footnotetext{
${ }^{2}$ Dans le tout premier rapport d'évaluation consacré à ces dispositifs, réalisé en 1996 par le CSERC pour le compte du Premier Ministre Alain Juppé, il était indiqué que sept secteurs de la nomenclature agrégée de l'époque, la NAP 40, étaient principalement concernés par les exonérations : les hôtels, cafés et restaurants ; les services marchands aux particuliers ; le commerce de détail, alimentaire et non alimentaire ; le textile et l'habillement ; les cuirs et chaussures ; enfin le bâtiment, génie civil et agricole. Il était indiqué également qu'un ciblage plus large des exonérations, jusqu'à 1,6 Smic, qui était le niveau maximal d'abattement retenu par la loi quinquennale pour l'emploi du 20 décembre 1993, ne modifierait qu'à la marge cette liste de secteurs. Plus récemment, les analyses descriptives de l'Acoss (2005) - Agence centrale des organismes de sécurité sociale -confirment ce point.

${ }^{3}$ L'indicateur d'hétérogénéité qui résulte du modèle de Malinvaud [2002] est la variance intersectorielle de la part du coût du travail non qualifié dans la valeur de la production. Plus les secteurs ont un contenu en emploi peu qualifié différents, plus
} 
macroéconomique risque bien de sous-estimer souvent la valeur absolue de l'élasticité à long terme de la demande de travail non qualifiée ».

Sans prétendre trancher cette question complexe du biais d'agrégation, l'objectif de cette contribution est d'évaluer les effets sur l'emploi des dispositifs d'exonération de cotisations sociales pour l'ensemble de l'économie et pour les secteurs les plus intenses en maind'œuvre. Pour y parvenir, nous avons construit un outil ad hoc d'évaluation ex ante, baptisé SISMICs, qui est un Simulateur Inter-Sectoriel pour la Mesure des Impacts des Cotisations Sociales. Cet outil très souple permet d'étudier les effets d'une modification du dispositif d'exonération sur le coût du travail et d'en déduire les effets directs et induits sur l'emploi pour l'ensemble de l'économie et pour les secteurs intensifs en main-d'œuvre

L'impact sur l'emploi des exonérations dépend en premier lieu de leurs effets sur le coût du travail et ces effets ne sont pas triviaux s'agissant d'un allègement dégressif qui s'applique à une distribution particulière de salaires ${ }^{4}$. L'impact sur le coût du travail résulte en effet de la rencontre entre un barème dégressif et une distribution des salaires qui est propre à chaque entreprise et à chaque secteur d'activité. Une première originalité de SISMICs est de proposer une mesure directe des effets des allègements de cotisations sociales sur le coût du travail selon différentes tranches de salaires définies relativement au Smic à partir des distributions effectives de salaires, au niveau des entreprises et des secteurs d'activité cohérentes avec les exonérations effectivement perçues. Cette distribution s'appuie sur des informations sur les salaires et le temps de travail tirées des DADS (Déclarations annuelles de données sociales) qui ont été calées afin d'obtenir des niveaux d'exonérations cohérents avec celles observées dans les fichiers de l'Acoss. Finalement, afin de préciser les effets indirects sur l'emploi des différentes réformes considérées, nous évaluons également l'ampleur des emplois induits au travers des échanges inter sectoriels de consommations intermédiaires.

Une deuxième originalité de cet outil est de pouvoir intégrer la dimension sectorielle afin d'insister sur le rôle joué par les secteurs intenses en main-d'œuvre. Alors que beaucoup d'études ont souligné dans une perspective de long terme les effets potentiellement négatifs des allègements sur la productivité et sur l'emploi qualifié (Chéron et alii, [2005] ; Langot [2011]), il s'agit de souligner les effets positifs des allègements sur l'emploi peu qualifié qui se concentrent dans un petit nombre de secteurs. Le dispositif français d'exonération de cotisations sociales mis en œuvre avec la réforme Fillon de 2003-2005 qui a harmonisé les dispositifs antérieurs hérités des mesures Aubry et des dispositifs Juppé du début des années quatre-vingt-dix, exerce des effets redistributifs entre les secteurs d'activité assez puissants au profit de ces secteurs intenses en main-d'œuvre. Dès lors, même une réforme marginale de ce dispositif, inspirée par la recherche d'économies de dépenses, dans un contexte de forte pression budgétaire, de croissance faible et de persistance du chômage à un niveau élevé, peut exercer des effets importants sur ces secteurs d'activité.

La dernière spécificité de cette étude est de mobiliser des élasticités de la demande de travail à son coût estimées sur des données d'entreprises ${ }^{5}$ (Bunel et L'Horty, 2012). Ces estimations ont été faites sur la période 2003 à 2007 au cours de laquelle le barème d'exonération a fortement évolué. Dans cette étude, nous évaluons les effets de plusieurs scenarii de réformes

\footnotetext{
l'on risque de sous-estimer les effets des allègements ciblés sur les travailleurs non qualifiés avec une approche agrégée (qui ne distingue pas plusieurs secteurs d'activité).

${ }^{4}$ Le lien entre exonération et coût du travail n'est d'ailleurs pas trivial non plus au niveau agrégé où les exonérations dégressives peuvent conduire à des effets inattendus des hausses du Smic sur le coût du travail (L'Horty, 2000).

${ }^{5}$ Niveau cohérent avec nos simulations.
} 
qui modifient un ou plusieurs paramètres du barème des exonérations (sa pente, son seuil d'extinction, son montant maximal, ou son système de décote avantageant les entreprises de moins de 20 salariés) Pour chaque réforme, nous évaluons les effets sur le coût du travail et les effets sur l'emploi pour l'ensemble de l'économie et pour les secteurs intensifs en maind'œuvre. L'organisation de cette étude est la suivante. La première section présente les dispositifs d'exonération de cotisations sociales et rappelle leur histoire. La deuxième section évalue les effets des exonérations sur le coût du travail et l'emploi pour ces entreprises. La troisième section explore des scenarii de réformes et mesure leurs effets sur le coût du travail et sur l'emploi. La dernière section conclut.

\section{LES DISPOSITIFS D'EXONERATION DE COTISATIONS SOCIALES SUR LES BAS SALAIRES}

La France est à la fois un pays où les prélèvements sociaux sont élevés, relativement à l'ensemble des autres prélèvements, en particulier l'impôt sur le revenu, et où les bas salaires sont élevés du fait de la présence du salaire minimum et de ses règles d'indexation, sur les prix à la consommation et le salaire moyen ouvrier.

La part des cotisations sociales payées par les employeurs dans le coût de la main-d'œuvre est plus importante en France que dans de nombreux pays industrialisés aussi bien au niveau global que lorsque l'on raisonne par sous-secteurs d'activité.

Dans ce contexte, les exonérations de cotisations sociales sur les bas salaires constituent un moyen de restaurer la compétitivité coût des entreprises. En l'absence de ces exonérations, le coût du travail au niveau du salaire minimum serait l'un des plus élevé d'Europe. Grâce aux allègements, la France est en cinquième position pour le niveau du coût du travail au salaire minimum.

Le dispositif qui organise l'exonération générale des cotisations sociales en France est issu de réforme du 17 janvier 2003, dite réforme Fillon, qui s'inscrit dans une longue tradition de dispositifs d'allègement de cotisations sur les bas salaires, ouverte en juillet 1993 avec les premières mesures du gouvernement Balladur. La réforme de 2003 consiste en la fusion de deux allègements existants, pour les entreprises aux 35 heures d'une part, et pour celles aux 39 heures d'autre part, en un unique nouveau barème, comme l'avait déjà effectuée la réforme de 1996 qui fusionnait l'exonération de cotisations famille de 1993 avec celle d'assurance maladie de 1995. De ce point de vue, l'exonération Fillon 2003 est dans la continuité des générations successives de dispositifs antérieurs qui ont progressivement approfondi l'ampleur des allègements tout en élargissant la cible vers des niveaux de salaires toujours plus élevés. Dans le même temps, la réforme Fillon a fusionné des dispositifs de salaire minima en organisant la convergence des mécanismes de Garantie Mensuelle de Rémunération, instaurées lors du passage aux 35 heures, et du SMIC (pour une évaluation des effets sur l'emploi de cette réforme, cf. Bunel et alii [2010] et Bunel et L'Horty [2012]).

\section{Une vue d'ensemble des exonérations générales}

S'il y a continuité dans l'empilement et l'extension des allègements, le dispositif Fillon marque aussi une rupture dans la longue succession de réformes des prélèvements sociaux. D'une part, il organise une uniformisation des barèmes des prélèvements sociaux selon les entreprises, qui colle au plus près des allègements Aubry II pour les salariés dont la rémunération est proche du Smic (le point de départ du nouveau dispositif reste à $26 \%$ ). D'autre part, les exonérations atteignent un maximum avec cette réforme. Le dispositif Fillon coïncide avec une montée en charge significative des exonérations de cotisations sociales qui vont représenter un montant de plus de 20 milliards d'euros à partir de 2007. Les premiers 
dispositifs mis en œuvre sous les gouvernements de MM. Balladur et Juppé représentaient quant à eux un montant budgétaire d'environ 5 milliards d' $€$.

Le graphique 1 présente les différents barèmes d'exonération de cotisations sociales employeurs qui ont été mis en œuvre en France depuis le début des années quatre-vingt-dix. On y a fait figurer également le dispositif en vigueur dans les départements d'outre-mer depuis 2009 (sur l'évaluation des effets sur l'emploi des exonérations outre-mer et sur les dispositifs antérieurs, cf. Bauduin et alii [2010] et [2011]).

\section{Graphique 1. Tous les dispositifs français d'exonération générale de cotisations sociales}

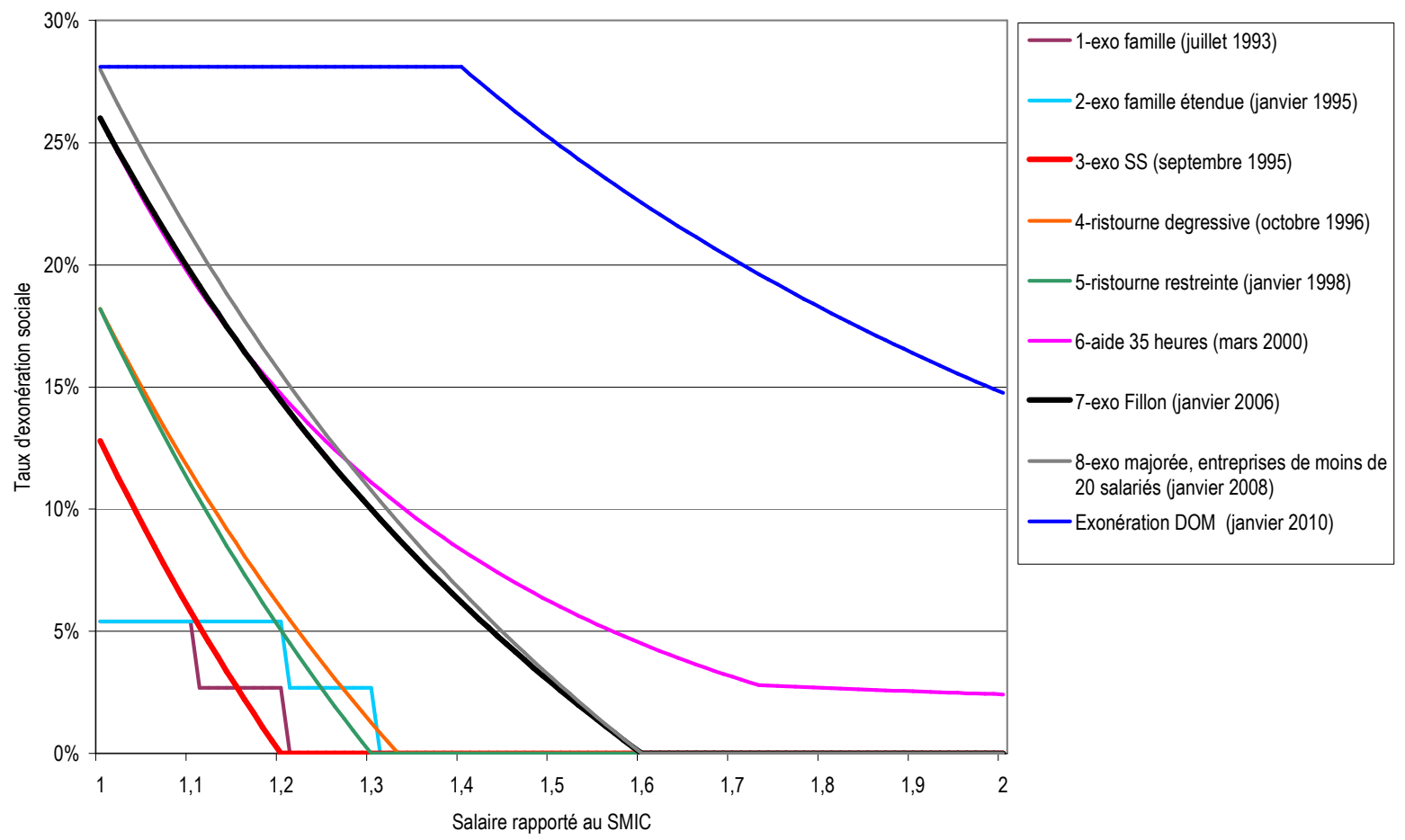

Source : Législation française et calculs des auteurs

Le volume de dispositifs qui dérogent au droit commun des prélèvements sociaux a connu une forte expansion en France sur les dernières décennies, à la fois en nombre de dispositifs et en masse budgétaire, sans que l'on puisse mesurer de façon précise cette expansion, tant les dispositifs sont nombreux et complexes. Un rapport du Conseil des Prélèvements Obligatoires (Amghar et Laloue, 2010) recense 68 dispositifs dérogatoires pour les mesures sur les salariés. Parmi tous ces dispositifs, 35 mesures affectent le taux de prélèvements à assiette constante (réductions de taux, franchises, exonérations...), 31 affectent l'assiette des prélèvements sans modifier le taux (non assujettissement, exemptions, abattement, déduction et plafonnement d'assiette).

Les allègements généraux gardent un poids prépondérant dans l'ensemble du budget consacré aux exonérations malgré l'accroissement important du nombre de dispositifs ciblés. Selon l'ACOSS [2011], les allègements généraux de cotisations sociales sur les bas salaires étaient de 20,9 Mds d'€ en 2010, soit 69,6\% de l'ensemble, tandis que les exonérations ciblées représentaient au total 6,1 Mds d' $€$ (soit $22 \%$ ), le solde correspondant aux exonérations pour heures supplémentaires (2,9 Mds d'€). S'agissant des seules mesures qui affectent les taux de prélèvement, trois grandes catégories de dispositifs dérogatoires peuvent être distingués : les 
exonérations sur les formes particulières d'emploi (apprentissage, contrat de professionnalisation, stages, contrat d'accompagnement vers l'emploi,...) représentent 2,2 Mds d'€, les exonérations territoriales (zones de redynamisation urbaine, zones de revitalisation rurale, zones franches urbaines, mesures DOM,...)représentent un budget de 1,3 Mds d' $€$, tandis que les aides sectorielles (franchise pour les services à la personne, jeunes entreprises innovantes, travailleurs occasionnels des secteurs agricoles, contrat vendange, marins salariés, portage de presse, arbitres et juges sportifs, journalistes professionnels, artistes du spectacle et mannequins, professions médicales, ...) représentent 2,6 Mds d' $€$.

\section{Brève histoire des allègements généraux de cotisations sociales}

L'ambition des premières mesures déployées en 1993 par le gouvernement d'Édouard Balladur était de baisser le coût du travail en général, et non d'agir uniquement sur le travail non qualifié. Avec la loi du 27 juillet 1993, qui instaure une exonération des cotisations familiales d'un niveau de 5,4\% entre le niveau du Smic et 1,1 Smic, et de 2,7 \% jusqu'à 1,2 Smic, on commence par cibler le bas de la distribution des salaires pour des raisons budgétaires. Mais une extension progressive des allègements jusqu'à 1,6 Smic était programmée par la loi quinquennale relative au travail, à l'emploi et à la formation professionnelle de décembre 1993. Elle a été mise en œuvre en partie à partir de janvier 1995 avec l'extension de l'exonération de cotisations famille jusqu'au seuil de 1,2 Smic (1,3 pour la demi-exonération).

Avec la loi du 4 août 1995, le gouvernement d'Alain Juppé instaure une ristourne dégressive des cotisations de Sécurité sociale. L'objectif n'était pas alors de baisser le coût du travail, mais d'éviter qu'il n'augmente après la hausse de $4 \%$ du salaire minimum de l'été 1995 qui a suivi l'élection présidentielle de Jacques Chirac. On parle aujourd'hui d'allègement défensif pour qualifier ce type de réforme visant à amortir les effets sur le coût du travail des hausses du Smic brut.

La réforme de 1996 poursuit quant à elle un objectif de simplification. Après les changements de 1993 et de 1995, deux dispositifs au profil différent coexistent, ce qui est inutilement compliqué et peu lisible. Le nouvel allègement correspond exactement à la somme des deux précédents au niveau du Smic $(18,2 \%=5,4+12,8)$ et reprend le profil linéairement dégressif du second dispositif. Le seuil d'extinction de l'exonération, qui est de 1,33 Smic, n'a pas été calculé de façon à optimiser les effets sur l'emploi non qualifié. Il a été simplement déduit de l'enveloppe budgétaire de 40 milliards de francs qui devait être affectée à cette politique, en supposant la distribution des salaires inchangée.

La réforme de 1998 réduit à 1,3 le seuil d'extinction de l'exonération. À nouveau, le changement n'est pas motivé par un souci d'optimisation des effets sur l'emploi. Il répond au besoin de dégager 4 milliards de francs d'économie budgétaire. Le nouveau seuil a été calculé de façon à être compatible avec une enveloppe de 36 milliards de francs. Le dispositif de 1998 est, par ailleurs, le premier à être calculé au prorata de la durée du travail, ce qui réduit l'avantage accordé au temps partiel.

Dans le cadre de la réduction de la durée collective du travail mise en œuvre par le gouvernement de Lionel Jospin, les allègements ont à nouveau un caractère défensif. Il ne s'agit pas de baisser le coût du travail, mais d'éviter qu'il n'augmente suite aux hausses des salaires horaires associées à la compensation de la baisse de la durée. Comme la compensation est intégrale au niveau du Smic et supposée partielle au-delà, l'allègement est dégressif avec les salaires, dans une fenêtre d'exonération élargie et selon un profil non linéaire. Ce profil légèrement convexe de la mesure dite «Aubry II » (2000) a été conçu afin 
de stabiliser le coût horaire à tous les niveaux de salaires, sur la base d'hypothèses pour les hausses de salaires horaires et celles de la productivité.

On retrouve les mêmes ingrédients dans la dernière réforme de 2003, dite « réduction Fillon ». Il ne s'agit toujours pas de baisser le coût du travail pour favoriser l'emploi non qualifié selon une logique offensive. L'objectif est, d'une part, de simplifier les dispositifs existants en fusionnant deux mesures qui coexistaient, comme en 1996. Désormais, un barème d'allègement unique s'applique, que les entreprises soient ou non à 35 heures. Il est d'autre part, d'éviter d'augmenter le coût du travail dans un contexte de forte hausse du Smic. Au niveau du salaire minimum, le nouveau dispositif est aussi avantageux pour les employeurs que celui de la mesure dite «Aubry II », et son profil est linéaire comme celui de la ristourne de 1998. Le surcroît de baisse de cotisations donné aux entreprises à 39 heures compense les effets sur le coût du travail de la hausse du Smic suite à l'harmonisation «par le haut » des mécanismes de garantie de revenus. Enfin, les disponibilités budgétaires ont déterminé le seuil d'extinction du dispositif, fixé à 1,7 Smic de façon transitoire, avant d'être porté à 1,6 Smic en 2005.

Deux réformes plus marginales ont été introduites. Celle de décembre 2006, visant à accroître de 2 points de pourcentage le seuil maximum d'exonération pour les entreprises de moins de 20 salariés. Celle de 2011 qui prévoit de calculer sur un base annuelle et non plus mensuelle les allègements versés afin d'éviter les éventuels effets pervers liés à la mensualisation identifiés dans le rapport Bur (2008).

Amortir l'impact sur le coût du travail des hausses de Smic ou des hausses de salaires, limiter les dépenses budgétaires, simplifier les dispositifs existants ont été les arguments à l'origine de chacune des réformes successives des dispositifs d'allègement de cotisations sociales. Aucune réforme n'a véritablement organisé une baisse offensive du coût du travail non qualifié dans le but de réduire le chômage des travailleurs non qualifiés. Pour autant, il n'en reste pas moins qu'au terme de toutes ces réformes, les gouvernements successifs ont fait le choix d'un dispositif original de réduction du coût du travail ciblé sur les bas et moyens salaires.

\section{Les principaux bénéficiaires des exonérations : les secteurs intenses en main-d'œuvre}

Les exonérations de cotisations sociales permettent certes de réduire le coût du travail pour les bas et les moyens salaires. Cependant, elles n'affectent pas de manière homogène le coût du travail supporté par les différents secteurs économiques. L'objet de cette section est d'identifier les secteurs d'activité qui bénéficient le plus des mesures d'exonération de cotisations sociales. Pour mesurer l'intensité de l'aide effectivement perçue, on utilise habituellement comme indicateur le taux d'exonération apparent (TEA) qui est le rapport entre le montant des exonérations et la masse salariale.

En 2010, ce taux est en moyenne de 5,7\% dans le secteur concurrentiel tous dispositifs d'aide confondus. Il inclut les exonérations générales, les exonérations géographiques et sectorielles spécifiques et les exonérations associées aux contrats aidés. Le TEA associés aux seules exonérations générales est de 4,9\%. La modification des barèmes d'allègement et la hausse du Smic associées à la réforme Fillon de janvier 2003 ont conduit à une progression de ce taux depuis 2003 où il a augmenté en moyenne de plus de $25 \%$.

Comme l'indique les travaux statistiques de l'Agence centrales des organismes de sécurité sociale (Acoss), ce taux est fortement influencé par la taille des entreprises (les plus petites bénéficiant d'un taux d'exonération plus élevé que les autres), la localisation géographique des entreprises et/ou leur secteur d'activité (Acoss-stat, 2005 ; 2011). 
Comme les allègements sont ciblés sur les bas et les moyens salaires (inférieurs à 1,6 Smic), ils bénéficient surtout aux secteurs riches en main-d'œuvre. Lorsque l'on calcule un niveau de TEA par sous-secteur d'activité, de nettes différences émergent. Ce taux est pratiquement nul ou marginal pour certains secteurs comme la $R \& D$ ou les activités financières alors qu'il est de plus de $11 \%$ pour les Hôtels et restaurants, les activités de services à la personne ou encore le commerce de détail.

Puisque les exonérations générales de cotisations sont décroissantes avec le niveau de salaire, plus les salaires sont élevés dans un secteur donné, moins ce secteur sera susceptible de bénéficier d'exonérations. Par exemple, le secteur de l'hébergement et restauration qui bénéficie du taux d'exonération apparent le plus important $(11,8 \%$ pour les seules exonérations générales) se caractérise par un salaire brut moyen mensuel $30 \%$ inférieur à celui observé dans l'ensemble du secteur concurrentiel.

\section{Graphique 2 : Taux d'exonération et part des salariés rémunérés en deçà de 1,3 Smic par secteur d'activité}

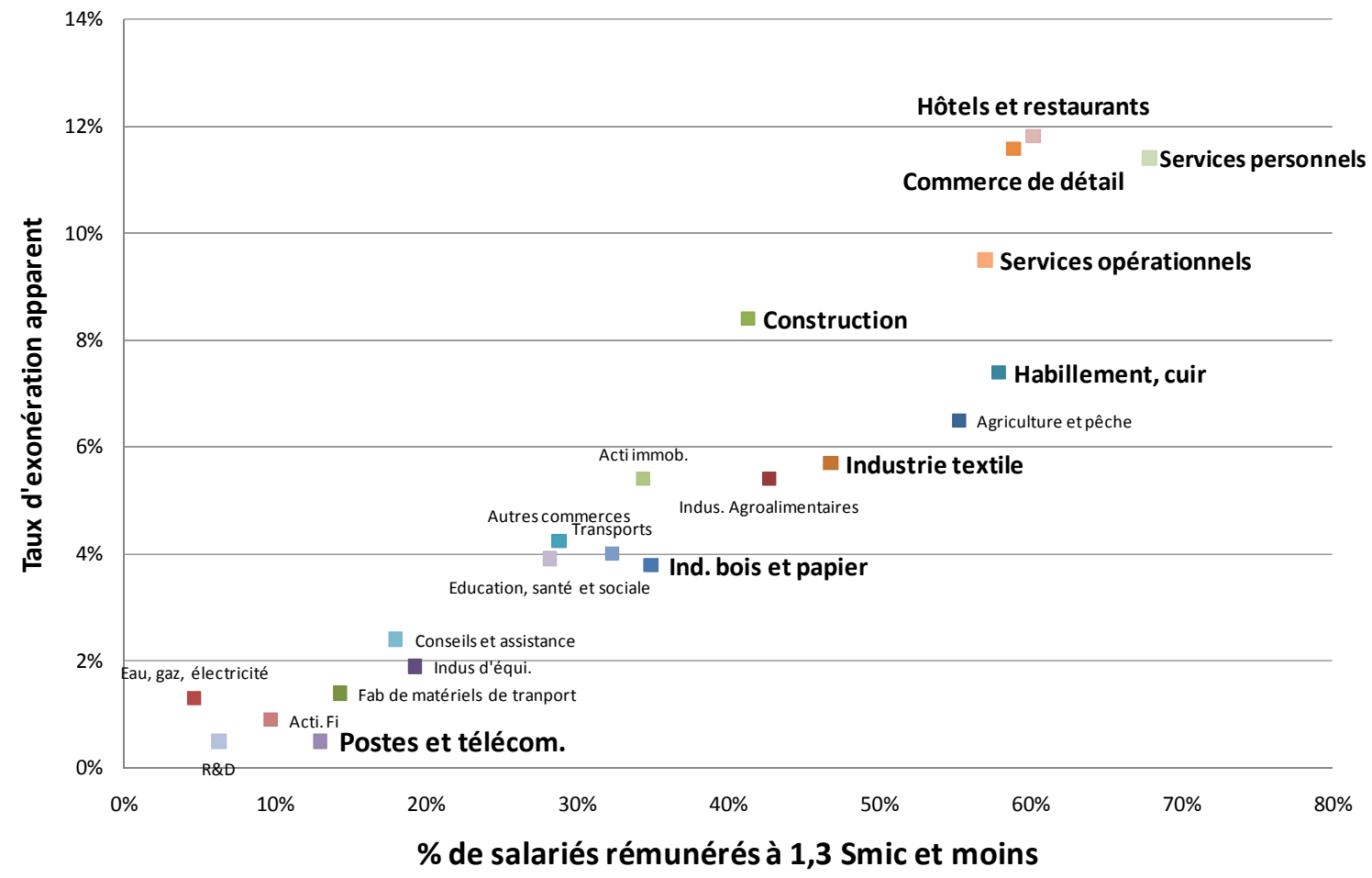

Source : Séquoia Acoss et Dads Insee - Calculs des auteurs

Cette relation entre niveau de salaire et taux d'exonération apparaît clairement sur le graphique 2. On observe un lien linéaire et positif entre la part des salariés rémunérés à 1,3 Smic et en deçà et le niveau du taux d'exonération apparent moyen par secteur. Ce graphique permet également d'identifier les secteurs qui bénéficient pleinement du système d'exonérations actuelles (situés en haut à droite) et ceux qui sont faiblement concernés (situés en bas à gauche). Les hôtels et restaurants, le commerce de détail, les services personnels et opérationnels, la construction et l'industrie textile sont les secteurs les plus concernés ${ }^{6}$.

\footnotetext{
${ }^{6}$ De même Heyer et al. (2012) montrent que l'introduction de la réforme de la TVA sociale proposée par le gouvernement Fillon en février 2012 et qui devait entrer en vigueur en octobre de la même année aurait un impact sectoriel très variables et favoriserait les secteurs (construction, agro-alimentaire et les transports) employant principalement des salariés rémunérés de 1,6 à 2,4 fois le Smic.
} 
Cette analyse sectorielle peut être complétée par une analyse géographique. Pour les mêmes raisons que celles avancées précédemment, les départements qui concentrent beaucoup d'entreprises à forte intensité en main-d'œuvre bénéficient également d'un niveau d'aide plus élevé. Les régions du centre et du sud de la France où les activités de services occupent une place importante sont celles qui en bénéficient le plus. En revanche, l'Ile-de-France, le RhôneAlpes et les régions du nord-est plus industrielles et/ou caractérisées par des niveaux de rémunération ${ }^{7}$ plus élevés se caractérisent par un taux plus faible de bas salaires et d'exonérations.

\section{SIMULER LES EFFETS DES EXONERATIONS SUR LE COUT DU TRAVAIL ET L'EMPLOI}

Afin d'évaluer les effets des exonérations de cotisations sociales, une maquette baptisée SISMICs (Simulateur Inter-Sectoriel pour la Mesure des Impacts des Cotisations Sociales) a été construite. Cet outil permet de calculer l'ampleur des aides perçues, leur impact sur le coût de travail par niveau de rémunération puis d'en déduire l'impact direct sur l'emploi.

Cette maquette permet également d'évaluer les effets indirects sur l'emploi. Plutôt que de proposer un bouclage macro-économique comme le font les autres évaluations (par exemple Heyer et al. (2012) à l'aide de la maquette e-mod.fr), SISMICs prend en compte les interdépendances sectorielles. Il est possible de quantifier le nombre d'emplois induits par des relais d'offre au travers des interdépendances productives entre secteurs d'activité.

Après un rappel des mécanismes théoriques, nous discutons des distributions de salaire intégrées dans la maquette. Le point suivant expose les effets des exonérations de cotisations sociales sur le coût du travail et les effets directs et induits sur l'emploi. Enfin, nous comparons nos résultats avec ceux obtenus par des travaux antérieurs.

\section{Les effets attendus en théorie}

Les exonérations générales de cotisations sociales poursuivent un objectif macroéconomique d'enrichissement du contenu en emploi de la croissance. Il s'agit de rendre le prélèvement social progressif en fonction du salaire, ce qui, à niveau donné de prélèvement, permet de réduire le coût relatif du travail peu qualifié et est favorable à l'emploi si l'on suit la théorie classique de la demande de travail. La hausse des exonérations de cotisations sociales sur les bas salaires, dès lors qu'elle n'est pas compensée par une hausse plus forte encore du salaire minimum et des salaires nets, peut réduire le coût du travail ce qui est susceptible d'exercer un effet positif sur l'emploi au travers de plusieurs canaux.

Au niveau microéconomique des décisions des employeurs, trois mécanismes sont en œuvre.

Le premier est dénommé effet de substitution : à niveau d'activité donné, la baisse du coût d'un facteur de production relativement aux autres facteurs va modifier les choix technologiques et organisationnels des entreprises, au détriment de l'emploi des autres facteurs de production, capital et travail qualifié. Cet effet peut aussi s'exercer entre les secteurs d'activités au travers de substitution dans la consommation : les secteurs les plus concernés par les exonérations diminuent leurs prix et captent une part plus importante de la demande.

> Le deuxième mécanisme est appelé effet de volume : à technologie et organisation donnée, la baisse du coût d'un des facteurs permet aux entreprises de baisser leurs prix ce qui peut stimuler la demande pour leurs produits et est favorable à l'emploi de tous

\footnotetext{
${ }^{7}$ Le coût horaire en Ile-de-France est de $43 \%$ plus élevé que dans les autres régions (Demailly et alii, [2012]).
} 
les facteurs de production. Ces deux premiers effets déterminent la valeur de l'élasticité de la demande de travail, c'est-à-dire la sensibilité de l'emploi demandé par les entreprises relativement au coût du travail.

Pour en déduire l'effet des exonérations sur le volume d'emploi, il importe également de prendre en compte un troisième mécanisme, que l'on appelle effet d'assiette. Pour une valeur donnée de la demande de travail, l'impact sur le niveau d'emploi d'une baisse de cotisation sociale est d'autant plus marqué que le coût du travail diminue fortement. Or, pour une enveloppe budgétaire donnée d'exonérations, la baisse du coût du travail va être d'autant plus forte en pourcentage que les exonérations seront ciblées sur les plus bas salaires. Cet effet d'assiette permet de renforcer les effets de volume et de substitution.

Formellement, le nombre d'emplois créé directement par une baisse de cotisations $\left(\boldsymbol{\Delta L}_{\boldsymbol{i}}\right)$ est égal au nombre initial d'emplois multiplié par l'élasticité de la demande de travail à son coût et par la baisse en pourcentage du coût du travail peu qualifié. Cette dernière est, pour un montant budgétaire d'exonérations $\boldsymbol{B}$ donné, d'autant plus forte que le salaire des travailleurs concernés est faible, c'est-à-dire que l'exonération est ciblée dans le bas de la distribution des salaires.

$$
\begin{aligned}
\Delta L_{i} & =L_{i} \frac{\Delta w_{i}}{w_{i}} \eta_{i}^{i} \\
\text { avec } \quad \frac{\Delta w_{i}}{w_{i}} & =-\frac{B}{L_{i} w_{i}}
\end{aligned}
$$

L'effet sur l'emploi est donc d'autant plus élevé que $\boldsymbol{w}_{\boldsymbol{i}}$ est faible, ce qui est d'autant plus le cas que la baisse de cotisations sociales $\boldsymbol{B}$ est ciblée dans le bas de la hiérarchie salariale. Cela reste valable quelle que soit la valeur de l'élasticité de la demande de travail, pourvu qu'elle soit bien négative.

Si les rendements d'échelle sont constants et si d'autres hypothèses standards sont respectées (iso-élasticité de la demande de biens, concavité de la fonction de production), l'expression théorique de l'élasticité de la demande de travail à son coût, issue du lemme de Shepard, est donnée par une formule à la fois simple et élégante.

$$
\eta_{i}^{i}=-\sum_{j \neq i} s_{j} \sigma_{j}^{i}-s_{i} \mathcal{E}
$$

Cette expression formelle de l'élasticité prix directe met en jeu les deux effets classiques de volume et de substitution qui vont dans le même sens. L'effet de substitution est d'autant plus important que le facteur dont le coût varie occupe une faible part de l'ensemble des coûts $\left(\mathrm{s}_{\mathrm{j}}\right.$ élevé) et que les possibilités de substitution entre facteurs sont fortes ( $\sigma$ élevé). L'effet volume est d'autant plus important que le facteur occupe une part importante dans l'ensemble des coûts ( $\boldsymbol{s}_{i}$ élevé) et que la demande est élastique aux prix ( $\boldsymbol{\varepsilon}$ fort). Notons que ces deux mécanismes jouent séparément l'un de l'autre et constituent donc deux canaux qui s'additionnent.

Ces effets favorables au niveau microéconomique peuvent être partiellement compensés à un niveau plus macroéconomique par d'autres mécanismes, appelés effets de bouclage. Par exemple, à long terme, les exonérations qui favorisent l'emploi peu qualifié peuvent aussi 
avoir des effets moins bénéfiques sur l'accumulation du capital humain, l'emploi des travailleurs qualifiés, la productivité et la croissance. Ensuite, si les exonérations augmentent l'emploi à court terme, elles vont contribuer à réduire le chômage ce qui peut favoriser une moindre modération salariale par effet Philips. Il en résulte une hausse de second tour des salaires qui modère la baisse initiale du coût du travail et limite l'impact positif sur l'emploi. En outre, les exonérations réduisent le coût relatif vis-à-vis de l'extérieur ce qui soutient la compétitivité-prix des exportations et réduit celle des importations, ce qui est favorable à la balance commerciale et à l'emploi. Enfin, le financement des exonérations a des conséquences sur l'équilibre des finances publiques. Pour compenser les manques à gagner de rentrées sociales, l'Etat doit réduire ses dépenses, emprunter ou augmenter les impôts ce qui suppose dans tous les cas un effet négatif sur l'emploi.

Il est important de souligner que les estimations que nous avons effectuées ne prennent pas en compte ces effets de bouclage macroéconomique. Elles se situent au niveau microéconomique et retracent les réactions des employeurs aux exonérations sans considérer les rétroactions possibles liées à l'équilibre général des marchés. En revanche, nous considérons les créations d'emploi induites par les interdépendances sectorielles. Les exonérations stimulent la production ce qui requiert des consommations intermédiaires qui augmentent la production dans les autres secteurs d'activité (cf. supra).

\section{La mesure des distributions des salaires}

Afin d'évaluer les effets des exonérations de cotisations sociales, il est nécessaire d'identifier clairement les salariés concernés et de connaître de façon précise leur niveau de rémunération. Cela suppose de positionner l'ensemble de salariés relativement au niveau du Smic. Une première difficulté est que les données administratives et statistiques françaises ne permettent pas de mesurer avec précision le nombre de salariés payés au Smic, comme le soulignent régulièrement les rapports du groupe d'experts sur le Smic (2009 à 2011). On peut seulement mesurer de façon relativement précise le nombre de salariés directement concernés par la revalorisation du Smic, à l'aide des enquêtes sur l'activité et les conditions d'emploi de la main-d'œuvre (Acémo) qui sont gérées par la Dares. Les fichiers administratifs des Déclarations annuelles de données de sociales (Dads) gérés par l'Insee conduisent quant à eux à identifier les salariés payés "au voisinage" du Smic. Cette approche ne correspond pas strictement à celle de l'assiette de vérification du Smic qui serait utilisée par un contrôleur du travail. Elle correspond aux salariés dont la rémunération annuelle perçue (incluant tout ou partie des éléments constitutifs du salaire) ramenée à l'heure de travail (calculée à partir du nombre de jours de paie et du nombre d'heures travaillées y compris les heures supplémentaires) est proche du Smic horaire légal.

Des écarts relativement importants peuvent être observés en mobilisant l'une ou l'autre de ces deux sources administratives. Par exemple, en 2006, d'après les fichiers Acémo, près de $11 \%{ }^{8}$ des salariés sont rémunérés au Smic dans les entreprises de 10 salariés et plus, alors que seulement $6 \%$ des salariés présents dans les Dads sont rémunérés au voisinage du Smic. Cet écart du simple au double s'explique par le fait que plus de la moitié des salariés rémunérés sur la base du Smic bénéficient d'une rémunération totale ramenée à l'heure de travail supérieure à 1,15 fois le Smic (Seguin, 2006). En effet, leur rémunération totale inclut des éléments faisant partie de l'assiette de vérification du Smic (voir annexe 1 pour une présentation détaillée de ces éléments) et des compléments exclus de cette assiette (primes d'ancienneté, primes liées aux contraintes de poste, majorations pour heures supplémentaires

\footnotetext{
${ }^{8} 15 \%$ toutes tailles d'entreprise confondues.
} 
ou complémentaires). Inversement, près de deux salariés sur 5 rémunérés au voisinage du Smic au sens des Dads ne sont pas repérés par l'enquête Acémo comme étant rémunérés au Smic. Le périmètre de ces deux fichiers ${ }^{9}$ ainsi que la mesure imparfaite du temps de travail expliquent cette erreur de type 2.

Ainsi, utiliser directement des données issues des Dads pour simuler les effets d'une modification des barèmes d'exonérations risque d'introduire un biais substantiel. La difficulté provient du fait que les Déclarations Annuelles de Données Sociales (Dads) constituent la seule source qui permet d'estimer pour chaque année la distribution des salaires horaires à un niveau sectoriel fin. En effet, les enquêtes Emploi disposent d'une taille d'échantillon trop faible pour pouvoir en déduire ce type d'information. L'enquête Acémo quant à elle reste peu accessible aux chercheurs et son champ exclut une partie des entreprises fortement utilisatrice d'emplois à bas salaire et de travail temporaire. L'enjeu est donc d'utiliser les Dads et d'essayer de corriger cette source pour tendre vers la vraie distribution des rémunérations.

Dans le cadre de la maquette SISMICs, on utilise les fichiers issus de la dernière année des Dads disponibles auprès de l'Insee (l'année 2008). Ces fichiers permettent d'établir une distribution brute des salaires en 11 classes pour l'ensemble de l'économie et pour les secteurs intenses en main-d'œuvre. D'après le graphique 3 qui présente cette distribution, $4,4 \%$ des salariés sont rémunérés au Smic en effectif occupé. Lorsque l'on tient compte de la quotité de travail moyenne des salariés de cette classe de rémunération sur l'année, ce pourcentage passe à 3,6\%. Ainsi, d'après cette source administrative, peu de salariés seraient rémunérés au Smic. On retrouve ici le biais identifié par les études antérieures.

\begin{tabular}{|l|}
\hline \multicolumn{1}{|c|}{ Les données DADS } \\
Les Déclarations Annuelles de Données Sociales (DADS) sont une formalité \\
administrative obligatoire pour toute entreprise employant des salariés. Le champ \\
de l'exploitation des DADS couvre actuellement l'ensemble des employeurs et de \\
leurs salariés à l'exception des agents des organismes de l'État (qu'ils soient ou \\
non titulaires); des services domestiques et des activités extraterritoriales. Les \\
DADS recouvrent donc en pratique $80 \%$ des emplois salariés. \\
Cette source statistique fournit des informations sur les salaires annuels bruts et \\
nets, le temps de travail, le type d'emploi, la catégorie socio-professionnelle et le \\
secteur d'activité. \\
Le temps de travail annuel s'obtient en croisant les informations sur le nombre de \\
semaines travaillées dans l'année et le nombre d'heures travaillées dans la \\
semaine. Cette mesure du temps de travail demeure relativement imprécise.
\end{tabular}

D'après cette distribution brute, $29 \%$ des salariés sont rémunérés en dessous de 1,3 Smic et $51 \%$ en dessous de 1,6 Smic. Ces pourcentages sont à considérer avec prudence et donnent une image vraisemblablement biaisée de la vraie distribution des salaires dans l'économie. Dans un travail antérieur visant à évaluer les effets sur l'emploi et les salaires de la réforme Fillon de janvier 2003, nous avons proposé une stratégie originale visant à caler la distribution brute issue des Dads en mobilisant une information administrative complémentaire : les montants d'exonérations de cotisations sociales effectivement perçus par les entreprises répertoriés par l'Agence centrale des organismes de sécurité sociale (Bunel, Gilles et l'Horty, 2010 ; Bunel et L'Horty, 2012).

\footnotetext{
${ }^{9}$ Par exemple, les entreprises agricoles, les entreprises de travail temporaire, les particuliers employeurs et les administrations publiques ne figurent pas dans le champ de l'enquête Acémo. De même, les apprentis, dont la rémunération minimale est définie comme une fraction du Smic, ne sont pas recensés parmi les salariés bénéficiaires de la revalorisation du Smic.
} 
Grâce aux bordereaux récapitulatif de cotisation (BRC) envoyés chaque mois pour les établissements de plus de 10 salariés et chaque trimestre pour ceux de moins de 10 salariés aux Urssaf, l'Acoss constitue un fichier Séquoia (Système pour l'Etude Quantitative et l'Observation des Assiettes) répertoriant l'ensemble des exonérations perçues par les établissements en les déclinant par types (principalement les exonérations générales, les exonérations spécifiques sectorielles ou territoriales et les exonérations associées aux contrats aidés).En collaboration avec les services de l'Acoss, cette base a été appariée entreprise par entreprise, avec les fichiers Dads. Ce travail a permis de comparer pour la première fois le niveau des exonérations effectivement perçues par les entreprises aux exonérations théoriques déduites de la distribution de salaire observée à l'aide des Dads. Un écart important a été identifié entre ces deux grandeurs. Le montant théorique issu de la distribution brute des salaires des Dads conduit à sous-estimer structurellement de l'ordre de $20 \%$ le montant des exonérations générales effectivement perçues.

Les secteurs intenses en main-d'ouvre
Secteurs intenses en main-d'œuvre correspondent aux secteurs concurrentiels, hors
agriculture et industries agroalimentaires, pour lesquels la part des emplois à bas
salaires (emplois rémunérés en deçà de la médiane des salaires définie à un niveau
NAF85) est supérieure à la part médiane de l'ensemble du secteur concurrentiel.
Les secteurs intenses en main-d'œuvre réunissent la quasi-totalité des emplois des
Hôtels et restaurants, des Services personnels, du commerce et de l'industrie
textile. A ces premiers secteurs, il faut ajouter entre $60 \%$ et $75 \%$ des emplois des
services opérationnels, de la construction, de l'industrie du bois et du papier et de
l'équipement, et des postes et télécommunication ${ }^{10}$. Enfin, une partie plus
marginale des emplois de la métallurgie et transformation des métaux et des
Transports est également intégrée. Il s'agit des sous-secteurs de la collecte des
déchets et de recyclage ainsi que des activités de joaillerie. Au total, 12 secteurs
sur les 36 sont considérés comme étant des secteurs intenses en main-d'œuvre, et
selon une intensité variable selon ces secteurs.
Ces secteurs intensifs en main-d'œuvre emploient au total 4,6 millions de salariés
dont 4,2 millions en équivalent temps plein. Ils représentent $31 \%$ des emplois du
secteur concurrentiel. Ces salariés bénéficient d'une rémunération plus faible que
la moyenne (-19\% en moyenne). La part de leur masse salariale dans la masse
salariale totale n'est que de $25 \%$.

Afin de corriger ce biais, cette distribution brute a été recalée pour qu'elle soit plus cohérente avec le montant des exonérations perçues réduisant l'écart final entre exonérations observées et théoriques à moins de $1,3 \%^{11}$. La distribution obtenue à l'aide de cette méthode et portant sur l'année 2008 figure dans le graphique 3. Cette opération conduit à des changements relativement conséquents. Par exemple, après calage 9,5\% au lieu de 4,4\% des salariés sont rémunérés au Smic dans le secteur concurrentiel. Ce résultat apparaît comme nettement plus

\footnotetext{
${ }^{10}$ Notons que ce secteur ne respecte pas, même à un niveau fin, la définition donnée aux secteurs intenses en main-d'œuvre. Il a toutefois été conservé dans ce champ car il comporte dans ses activités de distribution de courriers un taux de recours à des emplois à bas salaire très important.

${ }^{11}$ L'algorithme de calage utilisé est présenté en détails dans Bunel, Gilles et L'Horty (2010).
} 
cohérent avec les distributions récemment calculées à l'aide de l'enquête Acémo (Goarant et Muller, 2011 et annexe) $)^{12}$.

Notons que l'écart entre la distribution calée et la distribution brute est nettement moindre pour les salariés dont la rémunération est inférieure à $1,3 \mathrm{Smic}$ et à fortiori à $1,6 \mathrm{Smic}(34 \%$ contre $29 \%$ pour le premier groupe et $54 \%$ contre $51 \%$ pour le second). Ainsi, c'est bien aux alentours du Smic, c'est à dire pour des rémunérations inférieures à 1,2 Smic, que les Dads fournissent la distribution des salaires la plus biaisée.

Une fois ce calage réalisé, il est possible de comparer précisément les distributions de salaire entre le secteur concurrentiel et les secteurs intenses en main-d'œuvre (voir encadré).

Le graphique 4 présente les deux distributions obtenues. La distribution des salaires pour les secteurs intenses en main-d'œuvre est largement décalée vers la gauche : plus de $15 \%$ des salariés sont rémunérés au Smic (contre 9,5\% dans l'ensemble du secteur concurrentiel), 53\% touchent moins que 1,3 Smic (contre 34\%) et 75\% moins de 1,6 Smic (contre 54\%). Ainsi les trois quarts des salariés des secteurs intenses en main-d'œuvre seraient concernés par une modification des barèmes d'exonération contre moins de deux sur cinq dans les autres secteurs d'activité.

\section{Graphique 3 : Distribution des effectifs en fonction du Smic Ensemble de l'économie}

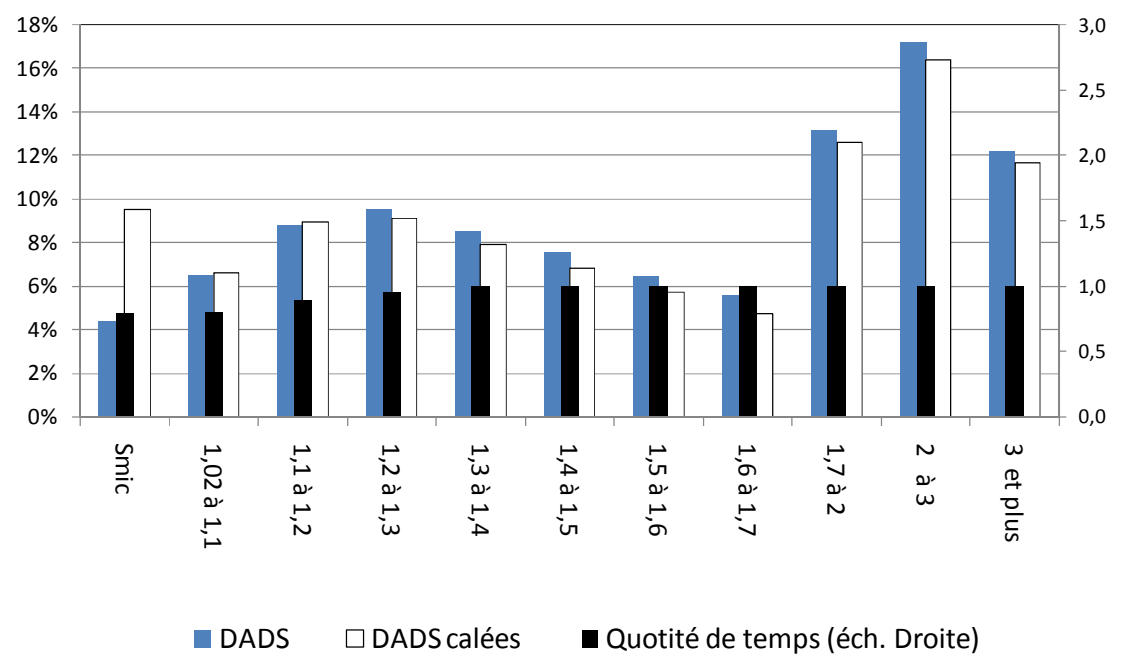

Source : Déclarations annuelles de données sociales, calculs des auteurs

\footnotetext{
${ }^{12}$ Pour le seul secteur HCR, un contrôle complémentaire du calage a pu être réalisé. En effet, il existait de 2004 à 2009 une aide spécifique à l'emploi dans ce secteur qui a conduit l'Unédic à constituer une base répertoriant le nombre de salariés au Smic parmi les salariés aidés. La distribution calée issue des Dads donne un nombre de salariés au Smic très proche de celui observé dans cette base (Bunel et L'Horty, 2011-b).
} 


\section{Graphique 4 : Comparaison des distributions des effectifs en fonction du Smic pour l'ensemble de l'économie et les secteurs intenses en main-d'œuvre}

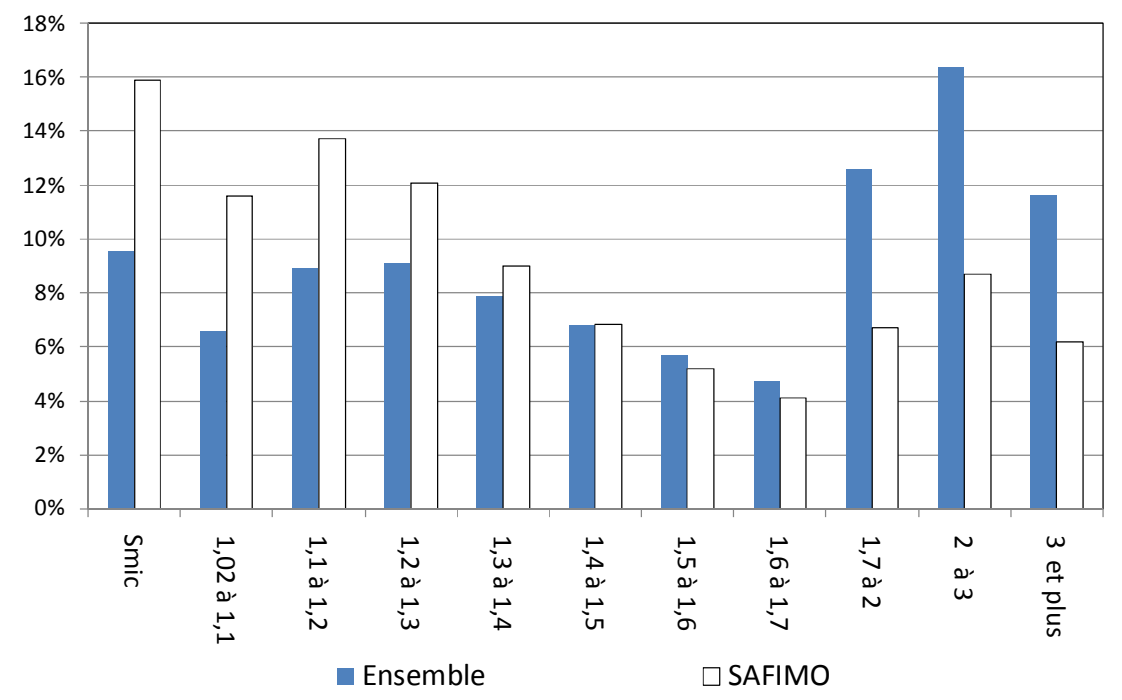

Source : Déclarations annuelles de données sociales, calculs des auteurs

\section{La mesure des effets sur le coût du travail et l'emploi}

A partir de la distribution sectorielle des salaires, il devient possible d'analyser les effets des exonérations générales sur le coût du travail et sur l'emploi. Il existe beaucoup de travaux d'évaluation dans ce domaine qui appliquent une pluralité de méthodes ex ante ou ex post, mais ces travaux portent tous sur la première génération d'exonérations, mise en œuvre avant 1998 et ciblée jusqu'à 1,3 Smic. Il existe peu d'évaluation des effets sur l'emploi du dispositif issu de la réforme Fillon de 2003 et ciblé jusqu'à 1,6 Smic. Comme les pentes des deux barèmes et leur degré de ciblage sur les bas salaires sont différents, on ne peut se contenter d'une simple règle de trois pour mesurer l'effet du nouveau dispositif. L'effet d'assiette et la baisse de l'élasticité de la demande de travail en fonction du niveau de rémunération font qu'un doublement du budget des exonérations conduit à des effets sur l'emploi inférieurs au double des effets initiaux si la fenêtre d'exonération est élargie et/ou si la pente du barème dégressif est plus faible. D'autres mécanismes économiques peuvent par ailleurs affaiblir le rendement des exonérations de cotisations au fur et à mesure de leur extension (décrits par Cahuc, 2003). Dès lors, il ne suffit pas de rapporter le coût budgétaire du dispositif actuel au coût par emploi créé issu des évaluations du dispositif antérieure, comme le font les chiffrages diffusés par l'administration économique (COE, 2006 ; Ourliac et Nouveau, 2012). Les destructions d'emplois déduites de cette opération sont comprises entre 600000 et 1,1 million d'emplois ce qui constitue un majorant de l'effet réel des exonérations générales.

Un travail plus poussé a été réalisé par Barlet et al (2009). La simulation proposée par ces auteurs intègre plusieurs mécanismes économiques notamment l'impact d'une variation de l'emploi sur le taux de chômage et la négociation salariale. L'ampleur des destructions d'emplois simulées à l'aide de la maquette ACE est nettement moindre. Elle est comprise entre 400000 et 600000 emplois sur 10 ans.

Notre objectif est de prolonger ces premiers travaux exploratoires en adoptant une stratégie sensiblement différente, de nature plus microéconomique. On s'appuie d'une part sur une mesure directe de l'effet des exonérations sur le coût du travail, obtenue par simulation des 
effets des barèmes sur les distributions de salaires. La mesure du coût du travail est déduite de la structure des barèmes et de la distribution des effectifs pour douze tranches de rémunération. D'autre part, on mobilise une estimation de l'élasticité de la demande de travail à son coût issue d'un travail micro-économétrique sur données d'entreprises portant sur la réforme des allègements de la période 2003 à 2005 (Bunel et L'Horty, 2012). L'élasticité moyenne de l'emploi au coût du travail que nous avons estimé est de 0,516.

Le tableau 1 présente les caractéristiques générales de l'ensemble des secteurs concurrentiels ainsi que des secteurs intenses en main d'œuvre qui ont été introduites dans la maquette SISMICs ainsi que les résultats des simulations.

Pour les secteurs intenses en main-d'œuvre l'effet des exonérations est nettement plus important. Les entreprises de ces secteurs bénéficient de près de 9 milliards d'euros d'exonérations générales de cotisations sociales par an. Ces exonérations représentent plus de $45 \%$ de l'enveloppe totale du secteur concurrentiel. Elles représentent 8 points de la masse salariale versée dans ces secteurs. Près de trois salariés sur quatre bénéficient dans ces secteurs du dispositif actuel d'exonération de cotisations sociales. Ce dispositif joue un rôle massif puisque pour ces salariés les exonérations réduisent de 14 points le coût du travail.

La maquette SISMICs met en cohérence ces différents éléments afin d'en déduire les effets de différents chocs, sur le coût du travail et sur l'emploi. Notre démarche qui consiste à chiffrer les effets sur l'emploi des exonérations générales par une simulation à partir d'une estimation de paramètres structurels, en l'occurrence l'élasticité de la demande de travail au coût du travail est celle suivie par Crépon et Desplatz [2001] et Gafsi et alii [2004].

Tableau 1 : Effets des exonérations actuelles pour l'ensemble du secteur privé et pour les entreprises des secteurs intenses en main-d'œuvre

\begin{tabular}{lccc}
\hline & & $\begin{array}{c}\text { Secteurs peu } \\
\text { intenses en main- } \\
\text { d'œuvre }\end{array}$ & $\begin{array}{c}\text { Secteurs intenses } \\
\text { en main-d'œuvre }\end{array}$ \\
\hline Effectifs occupés (en milliers) & Ensemble & 10185 & 4606 \\
Masse salariale (en milliards d'euros) & 14791 & 324,37 & 111,15 \\
Coût du travail par tête & 435,52 & $31848 €$ & $24128 €$ \\
\hline Exonérations simulées & $29444 €$ & & \\
\hline Cout des allègements de CS (milliards & & & 8,91 \\
d'euros) & 19,16 & 10,25 & 3424 \\
Nombre de salariés concernés par les CS (en & & & $74 \%$ \\
milliers) & 8078 & 4654 & $14,20 \%$ \\
Part des salariés concernés par les CS & $55 \%$ & $46 \%$ & $8,00 \%$ \\
Taux d'exonération moyen des exonérés & $12,34 \%$ & $11 \%$ & \\
Taux d'exonération moyen global & $4,4 \%$ & $3,2 \%$ & \\
\hline
\end{tabular}

Source : Déclarations annuelles de données sociales, maquette SISMICs

Deux variantes sont considérées. La première suppose que l'élasticité est constante pour tous les niveaux de salaire et de qualification. La seconde admet que cette élasticité est en moyenne de -0,516 mais qu'elle est plus importante pour les bas salaires et moindre pour les salaires plus élevés, comme le suggèrent des estimations de cette élasticité par niveaux de qualifications (Hamermesh, 1993).

Après simulations, nous trouvons que l'effet de l'ensemble des exonérations générales de cotisations sociales est compris entre 500000 et 610000 emplois créés ou sauvegardés (tableau 2). Une suppression pure et simple des allègements généraux de cotisations sociales 
conduirait à ce niveau de destruction d'emploi. Ces destructions se concentreraient fortement parmi les bas salaires : $35 \%$ au niveau du Smic et $85 \%$ pour des emplois compris entre le Smic et 1,3 fois le Smic. L'économie budgétaire réalisée par emploi détruit (ou coût par emploi créé) serait comprise entre 34000 et 42000 euros.

Quels seraient les effets directs de la suppression des exonérations générales sur les seules entreprises des secteurs intensifs en main-d'œuvre ? D'après les simulations réalisées à l'aide de la maquette SISMICs, l'effet serait de l'ordre de 236000 à 298000 emplois détruits soit près de la moitié des emplois détruits dans l'ensemble de l'économie alors que ces secteurs ne représentent que $30 \%$ de l'ensemble de l'emploi du secteur privé concurrentiel.

\section{Les créations d'emplois indirects}

A cet effet emploi direct il est nécessaire de le compléter par un effet induit de par l'interdépendance sectorielle. Pour ce faire, nous mobilisons le Tableau Entrées-Sorties (TES) national de l'année 2007 ventilé en 114 branches. Nous calculons sur cette base une matrice des coefficients techniques en divisant la valeur des consommations intermédiaires de chacun des produits par celle de la production de la branche, et ce, pour chaque branche. Cette matrice permet d'étudier les effets sur la production de toutes les branches de n'importe quel choc sur la production d'une ou plusieurs branches. La logique est celle des consommations intermédiaires requises pour la fabrication de nouveaux produits.

Pour passer des résultats exprimés en Production à des résultats exprimés en emploi, nous calculons le contenu en emploi de chaque euro produit pour chacune des 114 branches, à savoir $n_{i}=\frac{N_{i}}{P R O D_{i}}$ où $N_{i}$ est l'emploi régional par branche et $P R O D_{i}$ la production correspondante. Le coefficient $n_{i}$ est considéré comme étant stable au cours de l'année. Pour dénombrer les emplois induits, nous enlevons l'équivalent en production des emplois et regardons l'impact sur la production induite de l'ensemble des branches puis sur l'emploi induit.

Tableau 2 : Effets des exonérations générales sur l'emploi et coût par emploi créé

\begin{tabular}{lcc} 
Effet sur l'emploi (en milliers) & Ensemble & $\begin{array}{c}\text { Secteurs intenses en } \\
\text { main-d'œuvre }\end{array}$ \\
\hline élasticité fixe & 495,8 & 236,3 \\
élasticité variable & 613,0 & 298,2 \\
\hline Coût par emploi créé & & \\
\hline élasticité fixe & $42160 €$ & $41110 €$ \\
élasticité variable & $34090 €$ & $32580 €$ \\
\hline
\end{tabular}

Source : Maquette SISMICs

Les effets emplois ont été calculés de façon à neutraliser la couverture imparfaite des DADS.

La valeur de la production de la branche $i P R O D_{i}$ va s'ajuster au choc, $n_{i}$ restant constant. Cette variation $\triangle P R O D_{i}$ va provoquer à son tour des variations des productions des autres branches. Les coefficients $n_{i}$ des autres branches étant stables à court terme, le niveau de l'emploi requis $N_{i}$ dans chacune d'entre elles se modifiera. La somme de ces $\Delta N_{i}$ représente l'emploi induit. 
D'après cette simulation, une hausse de 100 emplois dans les secteurs intenses en maind'œuvre se traduit à terme par la création de 46 emplois induits. Dans ces emplois induits, 11 sont créés dans les secteurs intenses en main-d'œuvre. Ainsi, pour les 236000 à 298000 emplois détruits directement dans les secteurs intenses en main-d'œuvre suite à la suppression des exonérations, on devrait observer entre 108700 et 137200 emplois détruits indirectement dans l'ensemble de l'économie.

\section{Comparaison avec les études antérieures}

Il est intéressant de comparer ces résultats à ceux des évaluations antérieures, même si comme nous l'avons déjà précisé, on n'évalue pas le même dispositif. Dans le graphique 5A nous avons repris les résultats de 17 études antérieures en les ramenant à une enveloppe budgétaire de 5 milliards d'euro de 2010 (certaines études chiffrent les effets d'une baisse de 10 point de cotisations sociales, d'autres ceux de la mesure dite Juppé de 1995, d'autres encore celle d'un allègement de 10 milliards de francs). La moyenne des effets sur l'emploi est de 310000 emplois pour 5 milliards d'euros, ce qui est proche du budget des dispositifs des années quatre-vingt-dix. Pour ce budget, notre estimation moyenne est de 130000 emplois ce qui est inférieur mais néanmoins proche des résultats de plusieurs études. L'écart entre ces études peut provenir de deux effets.

D'une part, les premiers travaux portent sur les politiques d'exonération dont la pente et le ciblage sont plus favorables à l'emploi, à budget donné. D'autre part, les travaux sur données microéconomiques ont tendance à sous-estimer les exonérations effectivement perçues par les entreprises et ainsi à surestimer l'effet sur l'emploi. Le coût par emploi créé est selon nous compris entre $39000 €$ et $48000 €$, contre $24000 €$ en moyenne sur les 17 études antérieures qui ont évalué les mesures de première génération.

Graphique 5. Comparaison avec les études antérieures A - Nombre d'emploi créés ou sauvegardés pour une enveloppe de 5 milliards d'€

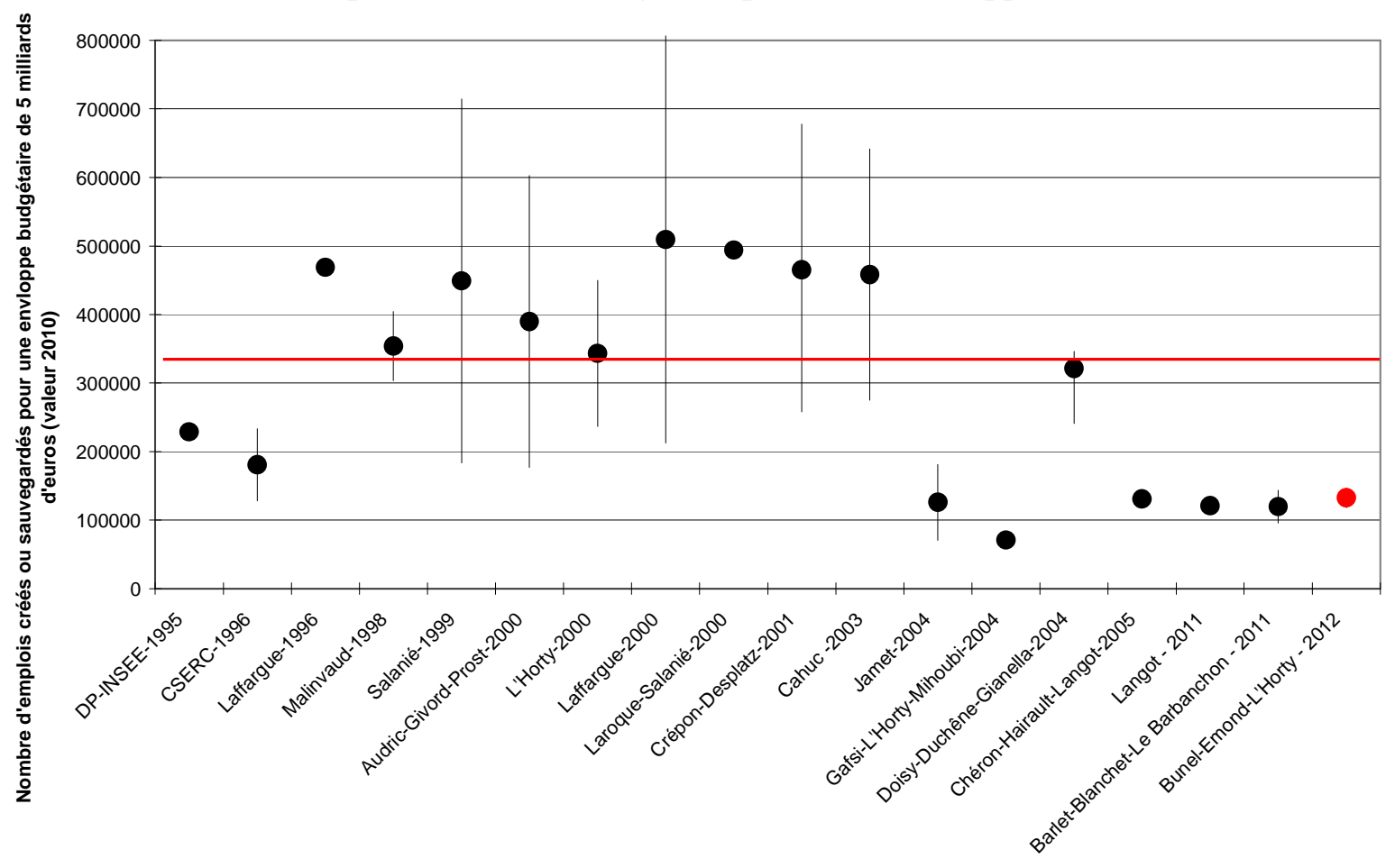


B - Coût par emploi créés ou sauvegardés

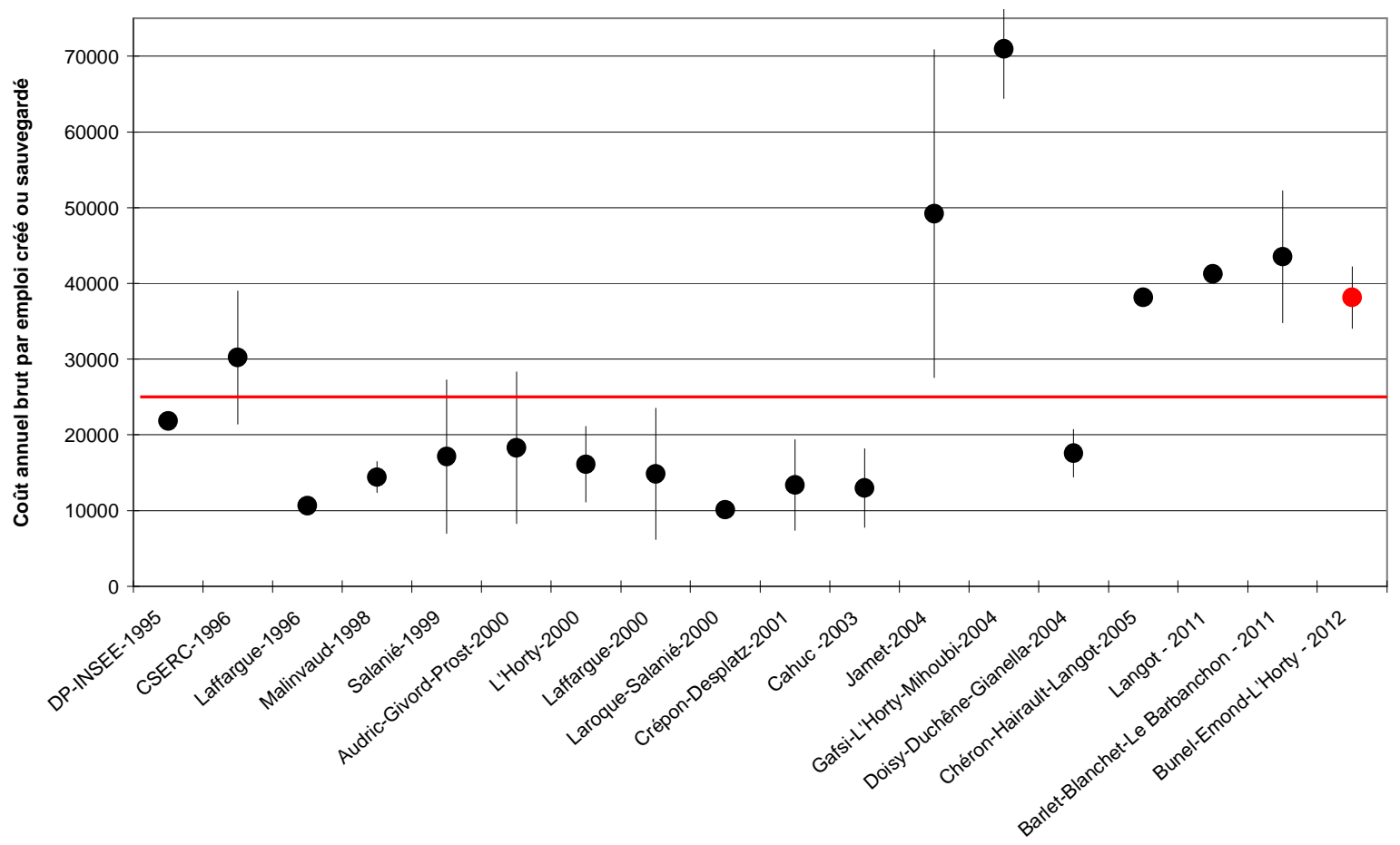

Source : calculs des auteurs

\section{LES EFFETS DE REFORMES PARTIELLES DES DISPOSITIFS GENERAUX D'EXONERATIONS}

La suppression totale des exonérations est une alternative qui a peu de chance d'être proposée et mise en œuvre à court terme. En revanche, des modifications des barèmes d'exonération peuvent être introduites conduisant ou non à une réduction de l'enveloppe totale d'exonérations.

Afin d'apprécier les effets potentiels de ces modifications sur l'emploi, nous avons simulé la mise en œuvre de plusieurs scenarii de réformes. L'idée est de balayer les possibles et d'étudier les effets sur l'emploi en focalisant l'attention sur les secteurs intensifs en maind'œuvre. Deux groupes de réformes sont appréhendées. Dans un contexte de forte pression budgétaire, on envisage en premier lieu des réformes visant à réduire d'un quart l'enveloppe totale des exonérations de cotisations sociales. Dans un second temps, on suppose que cette enveloppe reste constante mais avec un recentrage sur les bas salaires. Dans cette dernière configuration, on appréhende également les effets de la hausse du Smic.

\section{Six scenarii de réformes visant à économiser 5 milliards d'euros}

Nous avons imaginé six variantes de barèmes et mesuré leurs effets sur l'emploi et le coût du travail. Ces variantes modifient un ou plusieurs éléments caractérisant le système d'exonération actuel : sa pente ; son seuil d'extinction ; son montant maximal ; son système de décote avantageant les entreprises de moins de 20 salariés.

De façon à les rendre comparables, les paramètres des variantes ont été fixés de manière à pouvoir économiser exactement 5 milliards d'euros d'exonérations pour l'ensemble du secteur privé, soit une réduction de plus de $25 \%$ du total des exonérations. Présentons rapidement ces variantes, en les ordonnant de la moins ciblée sur les bas salaires à la plus ciblée : 
- Décotification : Ce scénario repose sur deux changements. Il consiste tout d'abord à supprimer le système de décote actuel portant sur le niveau maximum d'exonération de cotisations sociales. Ce seuil est de $26 \%$ et $28,1 \%$ selon la taille des entreprises (plus ou moins 20 salariés). Un nivellement par le haut de ce niveau à $29,90 \%$ pour toutes les entreprises est supposé. Les économies d'exonérations sont obtenues par une réduction du seuil d'extinction des exonérations à 1,35 Smic.

- Pentification : Ce scénario est plus simple et repose sur un seul changement la réduction du seuil d'extinction des exonérations. Ce seuil passerait de 1,6 à 1,4 Smic et il subsisterait un écart du niveau maximum d'exonération selon la taille des entreprises.

- Uniformisation : Ce scénario consiste à accroître pour l'ensemble des salariés y compris ceux rémunérés au de-delà de 1,6 Smic le montant des cotisations de 1,15 points de pourcentage.

- Translation : Cette réforme envisage un déplacement vers la gauche de la courbe des barèmes d'exonération pour l'ensemble des salariés. Les niveaux maximum d'exonération passeraient de $28,1 \%$ à $24,50 \%$ pour les entreprises de moins de 20 salariés et de $26,0 \%$ à $22,40 \%$ pour les autres. Le seuil d'extinction des exonérations serait réduit à 1,49 Smic.

- Sous-cotification : Ce cinquième scénario repose sur une modification du seuil d'exonération maximum des seules entreprises de 20 salariés et plus. Le seuil maximal baisserait sensiblement pour ces entreprises pour atteindre 15,30\%.Pour les plus petites entreprises, le barème d'exonération est supposé inchangé. En outre, pour l'ensemble des entreprises, le seuil d'extinction demeure figé à 1,6 Smic.

- Plafonnement : Le dernier scénario repose sur un plafonnement du niveau maximum des exonérations avec un maintien à $1,6 \mathrm{Smic}$ du seuil d'extinction des exonérations. Ce plafonnement serait de $17,1 \%$ pour les entreprises de 20 salariés et plus et 19,2\% pour les autres et affecterait tous les salariés jusqu'à 1,15 Smic.

En associant à chaque réforme une variation du coût de travail par tranche de salaire, il est relativement facile d'en déduire l'effet direct sur l'emploi. Les simulations réalisées indiquent que l'impact sur l'emploi varie entre 78000 et 166000 emplois détruits selon les scénarii (graphique 6A). Il faut dans tous les cas ajouter les emplois détruits indirectement qui représentent pour rappel 46\% des destructions directes soit entre 32700 et 69720 emplois. L'effet global sur l'emploi tenant compte des emplois directement détruits et des destructions induites varie entre 102700 et 235720 emplois.

La décotification qui consiste en un resserrement des exonérations sur les bas salaires avec une légère hausse du niveau d'exonération maximum apparaît comme la mesure qui affecte le moins le coût du travail et qui est la moins défavorable à l'emploi pour l'ensemble du secteur concurrentiel (moins de 80000 emplois seraient détruits dans ce cas).

Les graphiques 6A et 6B présentent l'ensemble des résultats des simulations réalisées pour les six réformes envisagées et en supposant soit une élasticité constante, soit une élasticité variable selon les niveaux de salaire. Le graphique 6-A porte sur l'ensemble de l'économie alors que le graphique 6-B se focalise uniquement sur les secteurs intenses en main-d'œuvre.

Les effets sur l'emploi obtenus sont cohérents avec l'évolution du coût du travail indiquée dans le tableau 3.Globalement, le classement de ces différents scénarii en fonction des destructions d'emplois respecte le principe suivant : les réformes de barèmes qui resserrent les exonérations vers les bas salaires sont celles qui génèrent le moins de 
destructions d'emplois. Inversement celles qui aplanissent le niveau des exonérations sont les plus défavorables à l'emploi.

Tableau 3. Six scénarii de réforme pour les exonérations générales de cotisations sociales : les paramètres d'intérêt

\begin{tabular}{|c|c|c|c|c|c|c|c|c|}
\hline \multirow{2}{*}{\multicolumn{2}{|c|}{ Scénarii et gains/coûts de la réforme }} & \multicolumn{3}{|c|}{ Paramètres d'intérêt } & \multirow[b]{2}{*}{ (1) } & \multicolumn{3}{|c|}{$\begin{array}{l}\text { Effet pour les secteurs } \\
\text { intenses en main- } \\
\text { d'œuvre } \\
\text { Variation du coût du } \\
\text { travail }\end{array}$} \\
\hline & & 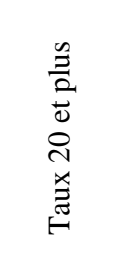 & 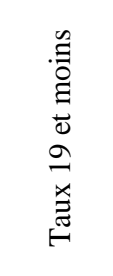 & 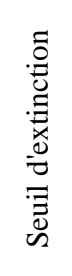 & & 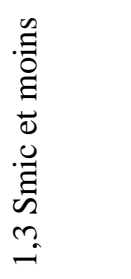 & $\begin{array}{l}\mathscr{\Xi} \\
0 \\
\Xi \\
0 \\
0 \\
.0 \\
\Xi \\
\tilde{\Xi} \\
0 \\
0\end{array}$ & 吾 \\
\hline $\begin{array}{c}\text { Décotification } \\
(-5 \text { milliards d' } €)\end{array}$ & 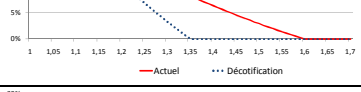 & $29,90 \%$ & $29,90 \%$ & 1,35 & $\begin{array}{c}-1,74 € \\
(34,8 \%)\end{array}$ & $1,2 \%$ & $2,4 \%$ & $1,3 \%$ \\
\hline $\begin{array}{c}\text { Pentification } \\
(-5 \text { milliards d' } €) \\
\end{array}$ & 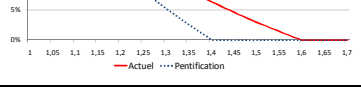 & $26,00 \%$ & $28,10 \%$ & 1,4 & $\begin{array}{c}-1,88 € \\
(37,6 \%)\end{array}$ & $2,1 \%$ & $2,6 \%$ & $1,4 \%$ \\
\hline $\begin{array}{c}\text { Uniformisation } \\
(-5 \text { milliards d' } €)\end{array}$ & \begin{tabular}{l|lll} 
& \\
\end{tabular} & $24,80 \%$ & $26,90 \%$ & 1,6 & $\begin{array}{l}-1,28 € \\
(25,6 \%)\end{array}$ & $2,1 \%$ & $2,0 \%$ & $1,9 \%$ \\
\hline $\begin{array}{c}\text { Translation } \\
(-5 \text { milliards d' } €)\end{array}$ & 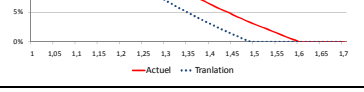 & $22,40 \%$ & $24,50 \%$ & 1,49 & $\begin{array}{c}-2,08 € \\
(41,6 \%)\end{array}$ & $3,2 \%$ & $2,9 \%$ & $1,5 \%$ \\
\hline $\begin{array}{c}\text { Sous-cotification } \\
(-5 \text { milliards d' } €)\end{array}$ & 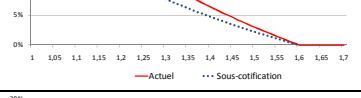 & $15,30 \%$ & $28,10 \%$ & 1,6 & $\begin{array}{l}-2,32 € \\
(46,4 \%)\end{array}$ & $4,6 \%$ & $3,2 \%$ & $1,7 \%$ \\
\hline $\begin{array}{l}\text { Plafonnement } \\
(-5 \text { milliards d'€) }\end{array}$ & "ox & $17,10 \%$ & $19,20 \%$ & 1,6 & $\begin{array}{c}-2,40 € \\
(48,0 \%)\end{array}$ & $4,9 \%$ & $3,3 \%$ & $1,8 \%$ \\
\hline
\end{tabular}

(1) Economie d'exonération supportée par les secteurs intenses en main-d'œuvre en milliards d'euros et en $\%$ du total.

Source : Maquette SISMICs 
Pour les secteurs intenses en main-d'œuvre des résultats similaires sont observés. Les destructions d'emplois dans ces secteurs varient entre 24000 et 84000 emplois selon les scénarii envisagés. Le plafonnement des exonérations apparaît à nouveau comme le changement le plus défavorable. Il conduit à la destruction de 3,5 fois plus d'emplois que la décotification.

Notons que le poids des destructions d'emplois supporté par les secteurs intenses en maind'œuvre change selon ces scénarii. Il est de l'ordre de $30 \%$ pour les trois premières hypothèses et varie entre $45 \%$ et $50 \%$ pour les trois dernières qui sont nettement plus défavorables aux bas-salaires.

Ainsi, dans l'optique d'une modification des barèmes d'allègement visant à économiser 5 milliards d'euros, la politique qui semble la moins défavorable à l'emploi consiste à réintroduire un ciblage des exonérations proche de celui établi par la réforme Juppé de 1995. Le montant maximum des exonérations demeurant nettement plus élevé que celui fixé il y a 17 ans.

Graphique 6 : Effet sur l'emploi des différents scénarii A- Ensemble de l'économie

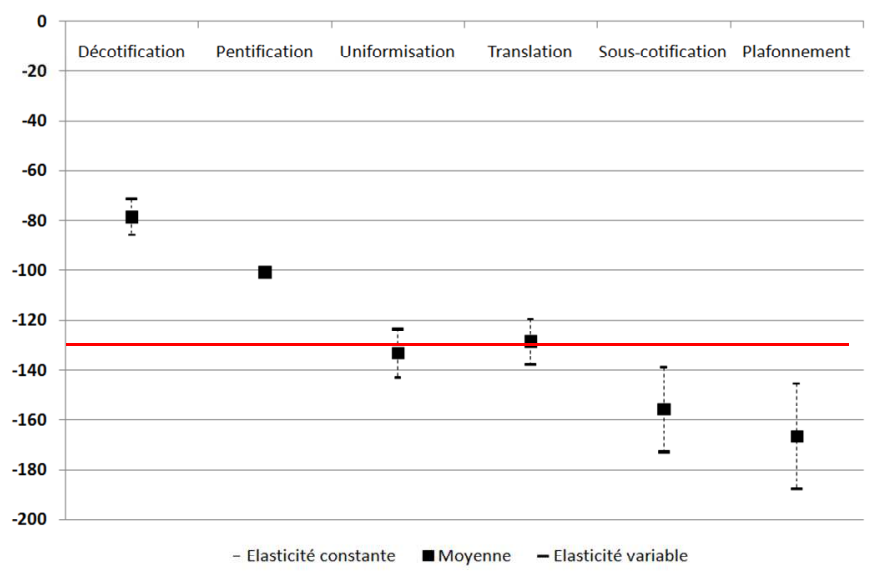

B- Secteurs intenses en main-d'œuvre

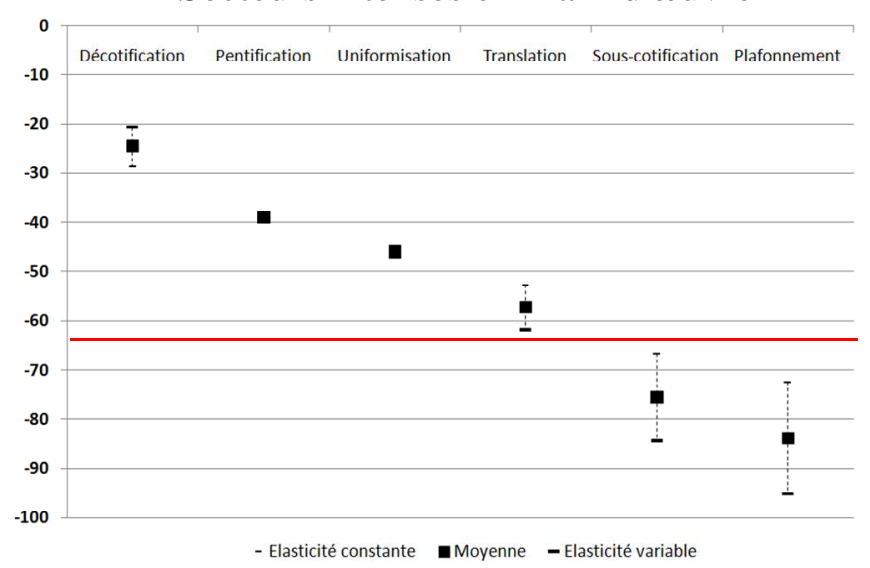

Note : les traits en rouge correspondent à l'effet moyen d'une suppression totale des exonérations de cotisations sociales ramenés à un coût de 5 milliards d'euros.

Source : Maquette SISMICs 


\section{Graphique 7 : Décomposition de l'effet emploi par niveau de salaire des différents scénarii}

A- Ensemble de l'économie

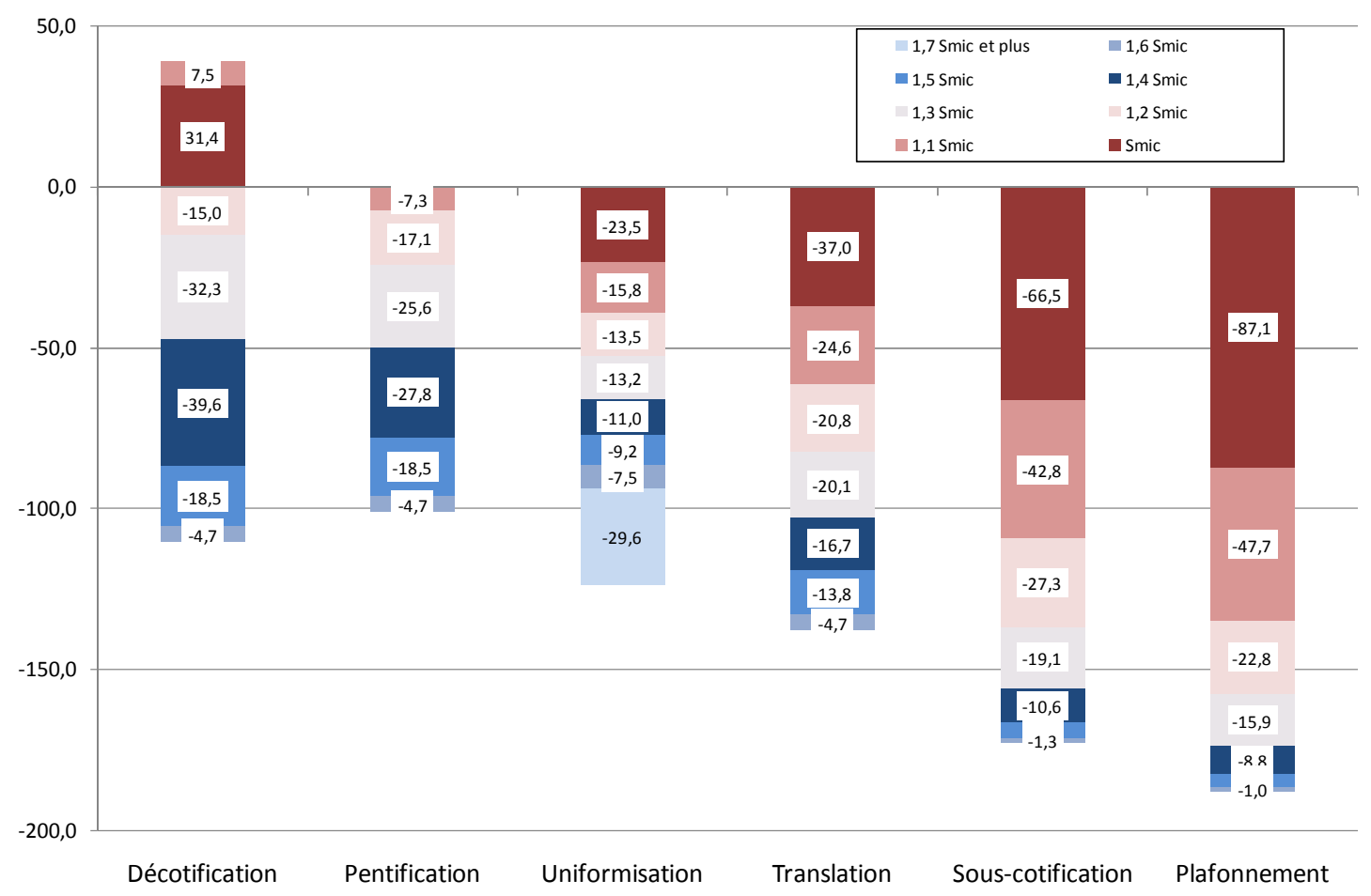

Source : Maquette SISMICs

B-Secteurs intenses en main-d'œuvre

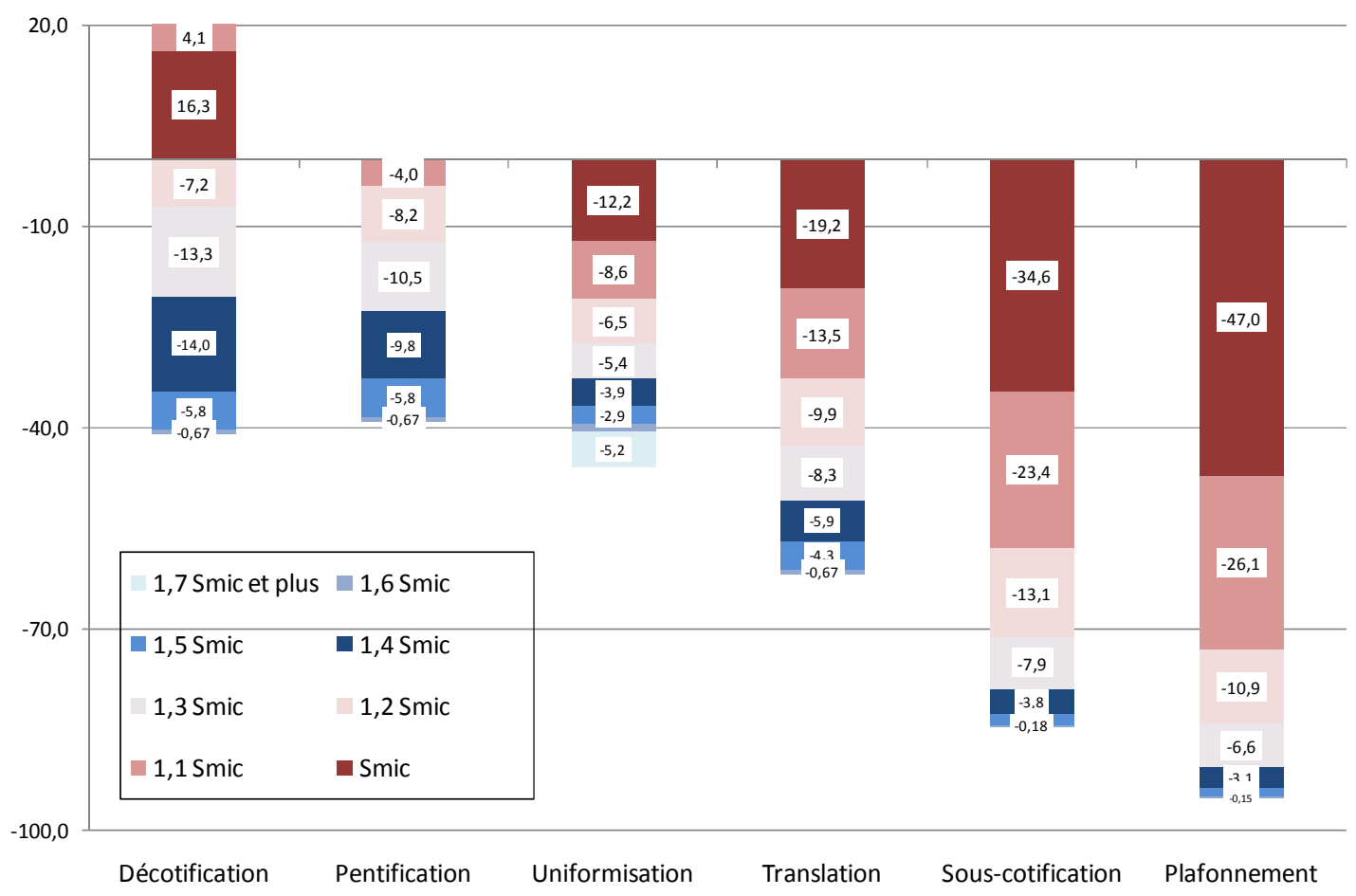

Source : Maquette SISMICs 
La question de l'émergence ou du renforcement d'une trappe à bas salaire dans le cas d'un tel ciblage reste ouverte. Les études qui tentent de mesurer l'incidence des exonérations générales sur la formation des salaires ne conduisent pas à des résultats univoques (voir notamment les travaux de Lehmann, Marical et Rioux, 2011 ; Lhommeau et Rémy, 2010 sur le sujet). Les travaux qui identifient l'existence d'une trappe à bas salaire montrent que son ampleur est relativement limitée (Lhommeau et Rémy, 2010).

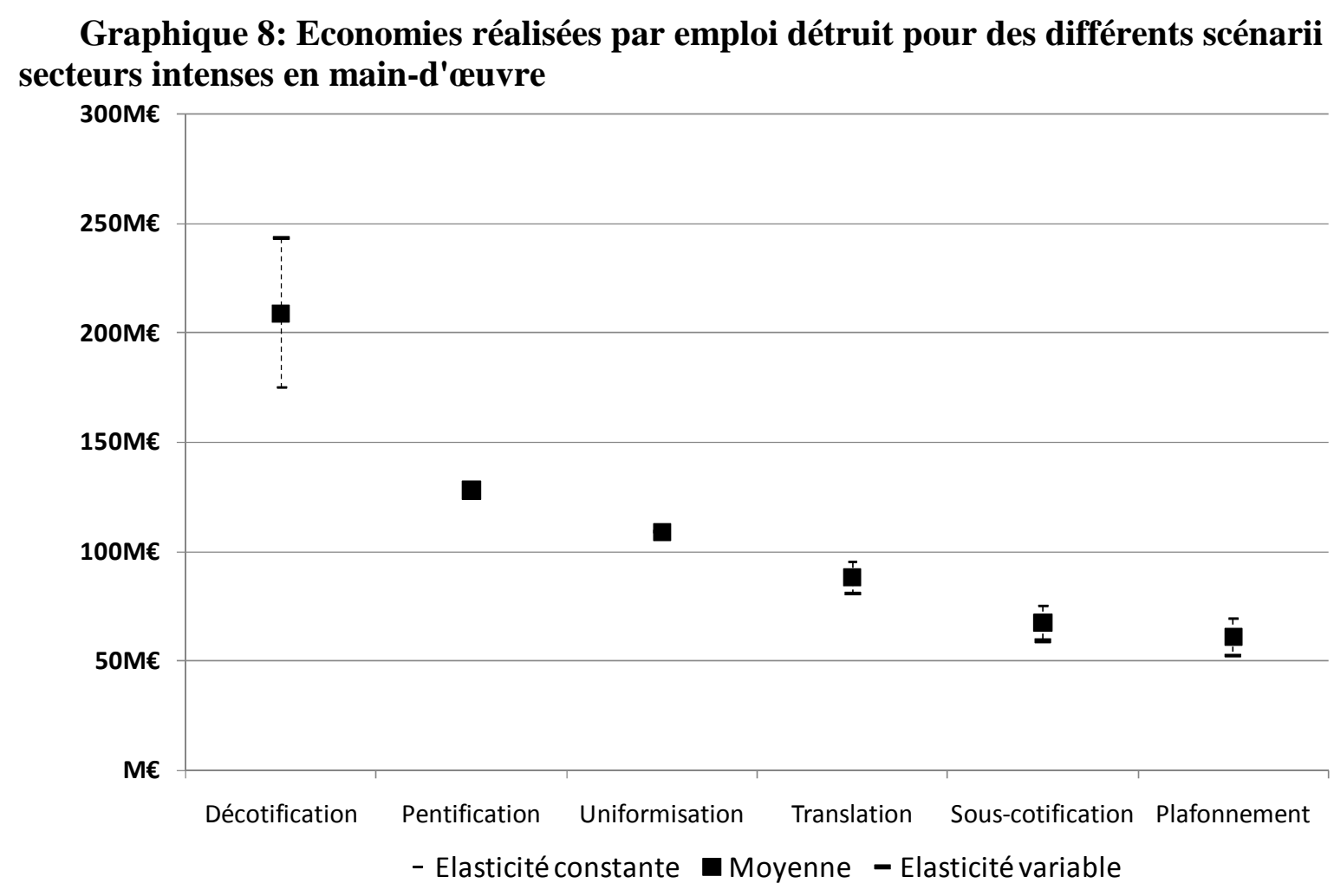

Source : Maquette SISMICs

La maquette SISMICs permet également de décomposer ces destructions d'emplois par tranche de rémunération (graphique 7A et 7B). Il apparaît qu'il existe une corrélation nette entre l'ampleur des destructions globales d'emplois et le nombre d'emplois à bas salaire détruits. Pour l'ensemble de l'économie, une réforme s'appuyant sur une décotification maintiendrait relativement inchangé le nombre d'emplois à bas salaires. Les 39000 emplois créés au voisinage du Smic seraient pratiquement compensés par les 47000 emplois détruits entre 1,1 et 1,3 Smic. Ainsi, l'essentiel des destructions d'emplois concernerait les salariés rémunérés au-delà de 1,4 Smic.

Inversement, pour les trois derniers scénarii, la translation, la sous-cotification et le plafonnement, entre $75 \%$ et $92 \%$ de ces destructions porteraient sur les bas-salaires.

Pour les secteurs intenses en main-d'œuvre, ce constat est renforcé, puisque seulement $1 \%$ des destructions d'emplois concerne les bas-salaires dans le cas d'une décotification alors que cette proportion varie entre $82 \%$ et $95 \%$ pour les trois derniers scénarii. Les réformes les moins défavorables à l'emploi sont celles qui sont les moins défavorables aux secteurs intenses en main-d'œuvre.

Finalement, le graphique 8 permet de donner des éléments de comparaison en termes d'efficience des mesures étudiées. Ce graphique exprime le montant des économies d'exonérations réalisées par emploi détruit. Par exemple, la décotification permet 
d'économiser en moyenne 210000 euros par an par emploi détruit alors que le plafonnement ne permet d'en économiser que 60000 euros. Globalement la première réforme apparaît 2 fois plus efficace qu'une politique de pentification ou d'uniformisation et 3 fois plus efficace qu'une politique de sous-cotification ou de plafonnement des exonérations. Ce ratio permet de hiérarchiser ces différentes réformes et de les comparer avec les résultats obtenus dans des études portant sur des chocs antérieurs (voir supra).

A titre d'information, il est également possible de calculer le coût par emploi sauvegardé associé aux exonérations qui demeureraient après ces réformes. Dans le cas d'une décotification, ce coût resterait sensiblement le même alors que dans le cas d'un plafonnement il augmenterait de près de $25 \%{ }^{13}$.

\section{Scenarii de réformes à budget d'exonérations constant}

Nous envisageons dans ce dernier point des réformes qui n'affectent pas le budget total des exonérations mais qui conduisent à les cibler davantage sur les bas salaires. Comme l'a montré le point précédent, la décotification et la pentification sont les réformes les plus efficaces en termes de maintien de l'emploi. C'est pourquoi nous ne retiendrons ici que des réformes affectant le seuil d'extinction et la pente des exonérations.

Nous envisageons deux variantes, ordonnée de la moins ciblée sur les bas salaires à la plus ciblée :

- Ciblage : Ce scénario repose sur deux changements la réduction du seuil d'extinction des exonérations et une hausse du taux d'exonération maximum. On suppose qu'il n'existe plus d'écart entre ce taux maximum selon la taille des entreprises.

- Ciblage avec plafonnement : Le scénario précédent conduit dans certaines configurations à obtenir un niveau maximum d'exonération qui dépasse le seuil de $28,1 \%$. Or la législation actuelle ne permet pas de dépasser ce seuil même si l'ensemble des exonérations de cotisations sociales dépasse $40 \%{ }^{14}$. Pour tenir compte de cette situation on introduit un plafond à $28,1 \%$. La forme du barème d'exonération s'apparente dans ce cas à celle existant dans les DOM.

\section{Graphiques 9. Deux scénarii types ciblant davantage les bas salaires à budget constant}

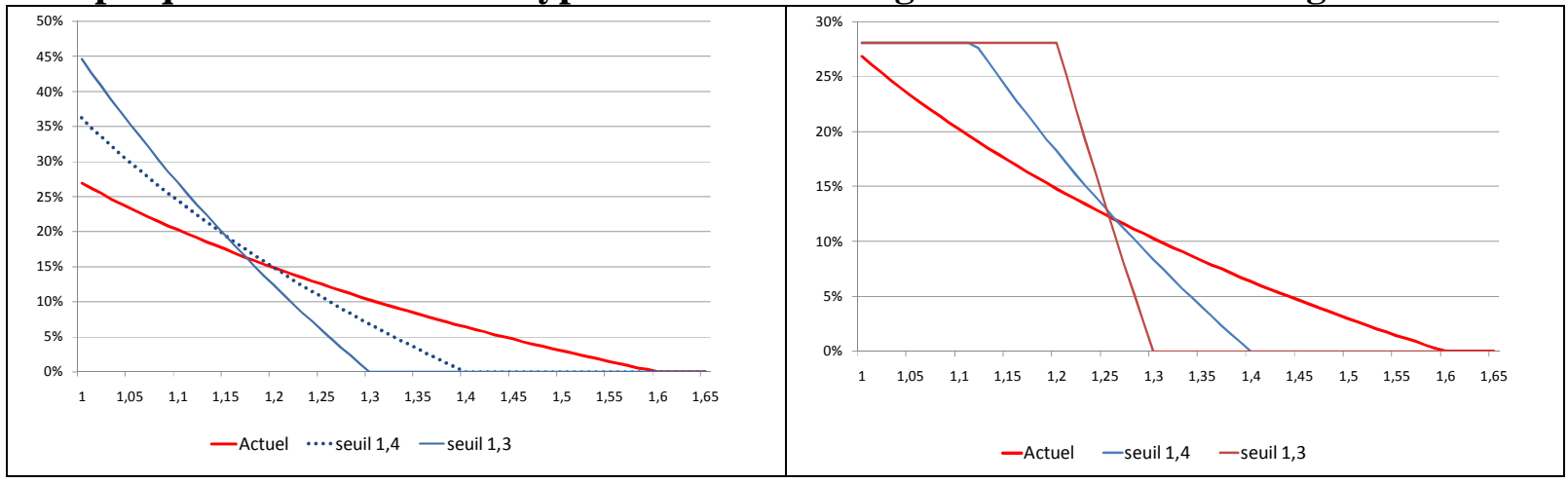

Source : Maquette SISMICs

\footnotetext{
${ }^{13}$ 6,507 milliards d'exonérations seraient toujours octroyées dans les secteurs intenses en main-d'œuvre pour un effet sur l'emploi de $(245,0-83,7)$ soit un coût par emploi sauvegardé de 40350 euros.

${ }^{14}$ L'ensemble des cotisations sociales patronales incluant notamment l'assurance chômage et les régimes complémentaires de retraite (AGIRC/ARRCO), est de prêt de 45\%. Or d'après la législation actuelle n'entrent pas dans le champ des exonérations les cotisations patronales d'assurance chômage, la contribution sociale généralisée (CSG) et la contribution au remboursement de la dette sociale (CRDS).
} 
Ce ciblage conduira à plusieurs effets. A titre d'exemple nous indiquons entre parenthèse les effets du passage du seuil d'extinction de 1,6 Smic à 1,3 Smic (sans plafonnement) :

1) Une part légèrement plus importante de l'ensemble des exonérations versées aux secteurs intensifs en main-d'œuvre (46\% à 49\%);

2) Un pourcentage moindre de salariés concernés par les exonérations (55\% à 34\%) ;

3) Une forte augmentation du taux d'exonération moyen (de 12,3\% à 23,3\% pour l'ensemble des entreprises et de $14,2 \%$ à $24,4 \%$ pour les secteurs intensifs en maind'œuvre) ;

4) Une baisse du coût du travail des salariés rémunérés en deçà de 1,3 fois le Smic (-4\%); Notons qu'en présence d'un plafonnement ces effets sont plus limités.

Le graphique 9 décrit les effets sur l'emploi d'une modification du seuil d'extinction. Un ciblage plus important des aides sur les bas salaires permettrait d'accroître le nombre d'emplois de 90000 à 170000 sans plafonnement et de 40000 à 55000 avec plafonnement des exonérations. Plus de $60 \%$ des emplois créés le seraient dans les secteurs intenses en main-d'œuvre.

\section{Graphique 10 : Effet sur l'emploi d'une modification du seuil d'extinction des exonérations à budget constant}

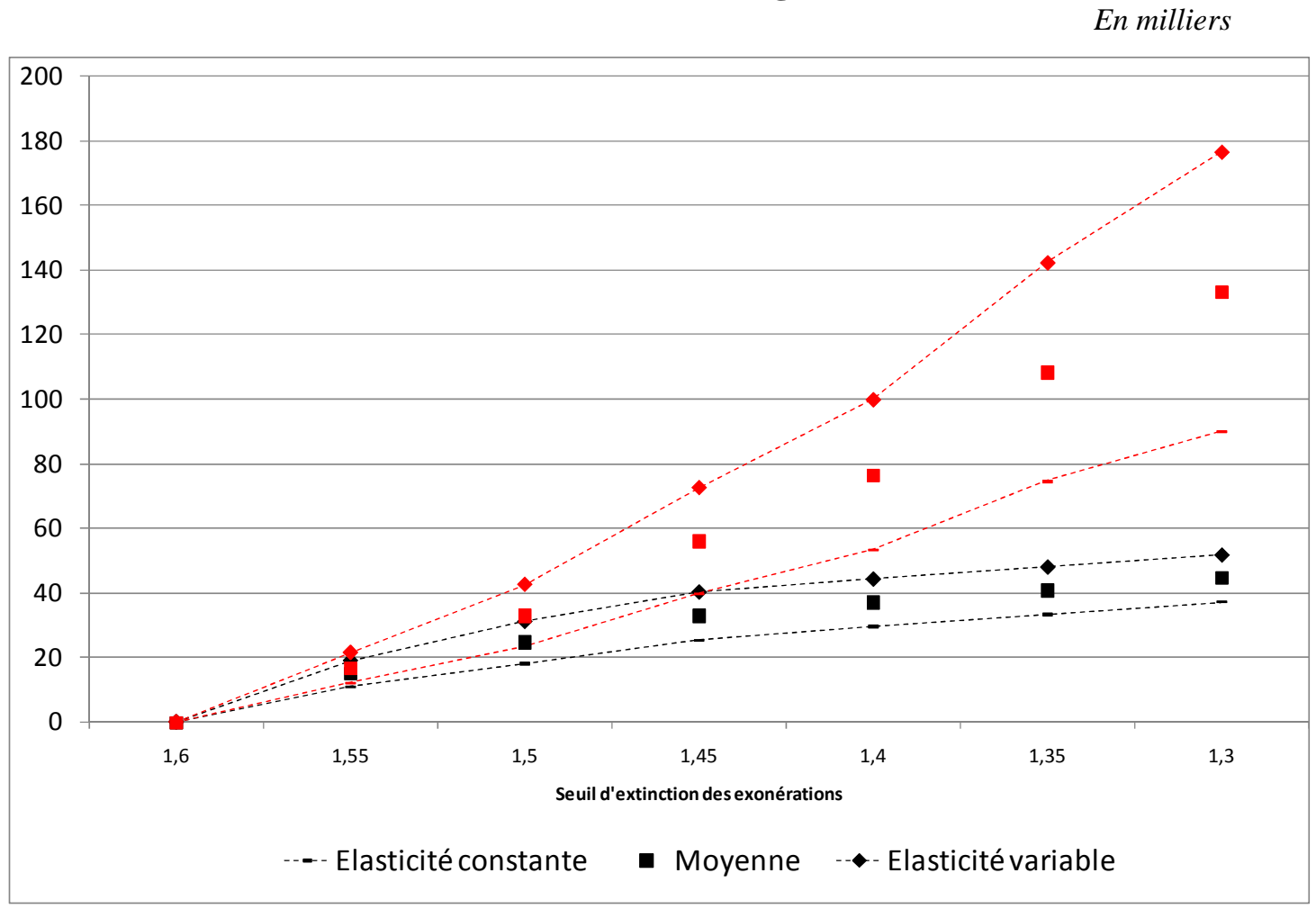

Lecture : les courbes en rouge correspondent à une modification du seuil d'extinction sans plafonnement, les courbes en noir supposent un plafonnement.

Source : Maquette SISMICs 


\section{Conclusions}

La politique française d'exonération générale de cotisations sociales sur les bas salaires est montée progressivement en puissance depuis les premiers dispositifs mis en œuvre en 1993.Elle a connu une extension marquée à la fin des années quatre-vingt-dix avec le passage aux 35 heures et une phase de stabilisation avec les mesures Fillon entre 2003 et 2005. Elle prend désormais la forme d'une exonération de 26 points de cotisations employeurs au niveau du SMIC, majorée à 28,1 points pour les entreprises de moins de 20 salariés, exonération qui diminue régulièrement avec le salaire jusqu'à s'annuler à $1,6 \mathrm{Smic}$. Ce dispositif mobilise un budget annuel de plus de 20 milliards d'euros, soit quatre fois plus que les budgets déployés au début des années quatre-vingt-dix. Grace à ces exonérations générales, la France n'est qu'en cinquième position européenne pour le niveau du coût du travail au voisinage du salaire minimum, alors que le niveau des prélèvements sociaux et celui du salaire minimum y sont parmi les plus élevés d'Europe.

Les exonérations de cotisations sociales sur les bas salaires ont un effet incontestablement positif sur l'emploi, comme l'ont confirmé de façon unanime les 17 évaluations déjà réalisées. Mais ces travaux portent tous sur les dispositifs de première génération mis en œuvre au début des années quatre-vingt qui étaient très ciblés sur les bas salaires (avec un seuil d'extinction à 1,3 Smic), à l'exception de l'évaluation ex ante de Barlet et alii (2010). En outre, ils ne prennent pas en considération la dimension sectorielle des dispositifs d'allègements généraux. Pour une entreprise donnée, le montant des exonérations dépend fortement de la distribution des salaires, qui renvoie pour l'essentiel à la structure de ses qualifications. Les secteurs d'activité les plus riches en main-d'œuvre sont donc les plus concernés par ces dispositifs, tandis que dans d'autres secteurs plus intenses en travail qualifié et en capital, il n'y aura pas ou peu d'effets sur le montant des prélèvements sociaux à la charge des employeurs.

Les exonérations générales de cotisations sociales représentent $5,7 \%$ de l'ensemble de la masse salariale des salariés du secteur privé en 2010. Ce taux moyen étant très variable selon les secteurs d'activité. Un petit nombre de secteurs est principalement concerné par les exonérations. Il s'agit des hôtels et restaurants, des services à la personne, du commerce, de l'industrie textile, des services opérationnels, de la construction, de l'industrie du bois et du papier et de l'équipement, et des postes et télécommunication. Il s'agit également des soussecteurs de la collecte des déchets et de recyclage ainsi que des activités de joaillerie. Ces secteurs intenses en main-d'œuvre emploient dans l'ensemble 4,6 millions de salariés et 4,2 millions en équivalent temps plein. Ils représentent $31 \%$ des emplois de l'ensemble des secteurs concurrentiels. En moyenne, leurs salariés bénéficient d'une rémunération plus faible $(-19 \%)$. C'est pourquoi, la part de leur masse salariale dans la masse salariale totale n'est que de $25 \%$.

D'après nos simulations, dans ces secteurs intenses en main-d'œuvre près de trois salariés sur quatre bénéficient des exonérations ce qui conduit ces secteurs à capter plus de $45 \%$ du budget dédié aux exonérations générales de cotisations sociales. Globalement, ce dispositif représente 8 points de la masse salariale versée dans ces secteurs et réduit de 14 points le coût du travail des salariés rémunérés en deçà de 1,6 fois le Smic.

L'objectif premier de notre étude était d'évaluer les effets sur l'emploi des exonérations générales de cotisations sociales pour l'ensemble de l'économie et pour les secteurs les plus intenses en main-d'œuvre. Pour cela, nous avons construit un outil ad hoc d'évaluation ex ante, baptisé SISMICs, qui est un Simulateur Inter-Sectoriel pour la Mesure des Impacts des Cotisations Sociales. Cet outil nous a permis d'étudier les effets sur le coût du travail et sur 
l'emploi d'une suppression totale du dispositif d'exonération générale de cotisations sociales existant et de plusieurs réformes visant à l'aménager en partie. Ces réformes sont plus ou moins ciblées sur les bas salaires et sont analysées pour une enveloppe budgétaire fixée arbitrairement à 5 milliards d'euros. Pour chaque réforme, nous évaluons les effets sur le coût du travail et les effets sur l'emploi en partant d'élasticités de la demande de travail à son coût estimées sur des données d'entreprises, c'est-à-dire à un niveau microéconomique cohérent avec nos simulations. L'originalité de cette maquette est de prendre en considération la distribution réelle des emplois, selon différentes tranches de salaires définies relativement au Smic. Cette distribution s'appuie sur des informations sur les salaires et le temps de travail tirées des DADS (Déclarations annuelles de données sociales) qui ont été calées afin d'obtenir des niveaux d'exonérations cohérents avec les données de l'Acoss. Afin de préciser les effets indirects sur l'emploi des différentes réformes considérées, nous évaluons également l'ampleur des emplois induits au travers des échanges inter sectoriels de consommations intermédiaires. L'étude a été complétée par l'examen de cas-types d'entreprises intenses en main-d'œuvre qui montrent qu'en matière d'exonérations générales, des réformes uniformes ont des effets très différenciés selon les entreprises (voir annexe 3).

D'après les résultats de nos simulations, la suppression de l'ensemble des exonérations générale de cotisations sociales conduirait à une hausse du coût du travail de l'ordre de 4,4\% pour l'ensemble des salariés et de $12,3 \%$ pour les salariés qui bénéficient à l'heure actuelle des exonérations. Les destructions d'emplois associées à ce renchérissement du coût du travail seraient comprises entre 500000 et 610000 emplois dont près de la moitié dans les secteurs intenses en main-d'œuvre. Les pertes d'emploi dans les secteurs de main-d'œuvre conduisent en outre à des pertes d'emplois induites dans les autres secteurs qui correspondent à $46 \%$ des pertes initiales (100 emplois détruits dans les secteurs intenses en main-d'œuvre détruisent indirectement 46 emplois dans le reste de l'économie). Les destructions d'emplois directs se concentreraient fortement parmi les bas salaires : 35\% au niveau du Smic et $85 \%$ pour des emplois compris entre le Smic et 1,3 fois le Smic. L'économie budgétaire réalisée par emploi détruit (ou coût par emploi créé) serait comprise entre 34000 et 42000 euros.

Nous avons tout d'abord imaginé et simulé six variantes de barème, pour une économie budgétaire donnée de 5 milliards d'€, qui modifient un ou plusieurs éléments caractérisant le système d'exonération actuel (sa pente ; son seuil d'extinction ; son montant maximal ; son système de décote avantageant les entreprises de moins de 20 salariés). Ces réformes sont plus ou moins ciblées sur les plus bas salaires et sur les secteurs de main-d'œuvre. La part de la baisse des exonérations à la charge des seules entreprises des secteurs intenses en maind'œuvre varie du simple au double. Les résultats des simulations indiquent que les réformes les moins défavorables à l'emploi, sont celles qui préservent le plus les bas salaires et les entreprises qui les emploient. Nous avons également imaginé un ciblage des exonérations à budget constant permettant de renforcer très nettement l'ampleur des exonérations versées aux salariés rémunérés en deçà de 1,3 Smic. Cette politique permettrait de créer plusieurs dizaines de milliers d'emplois à coût constant et ceci principalement dans les secteurs intensifs en main-d'œuvre. La question complexe du développement dans un tel contexte d'une trappe à bas salaire reste ouverte.

Les spécificités et les limites de notre travail doivent être indiquées. Il s'agit d'une évaluation qui utilise les distributions de salaires effectives des entreprises et leurs comportements d'emploi estimés sur micro-données administratives. Nous supposons une élasticité de la demande de travail de 0,5 en moyenne, éventuellement décroissante avec le niveau de qualification, qui n'aurait pas été affectée par la crise économique. Cette évaluation ne prend pas en compte les effets d'entraînement entre secteurs d'activité mais n'intègre pas d'effets de bouclage macroéconomique par la formation des salaires, celle des prix, l'équilibre extérieur 
ou celui des finances publiques. Ces interdépendances macroéconomiques ont largement été documentées par des travaux antérieurs et paraissent peu pertinent a priori pour une discussion sur le ciblage optimale des aides.

\section{Références bibliographiques}

Acoss (2011), «En 2010, les exonérations se stabilisent », Acoss-stat 138, novembre 2011.

Acoss (2005), «Les exonérations de cotisations sociales en 2004 : forte hausse des petits établissements», Acoss-stat 33, décembre 2005.

Amghar Y-G et Laloue, (2010), Les dispositifs dérogatoires en matière de prélèvements sociaux, rapport du Conseil des Prélèvements Obligatoires, Juillet 2010.

Artus P., (2012), «Quelques points importants à prendre en compte avant de faire des recommandations de politique économique dans les pays de la zone euro », Eco Flash, 20 janvier 2012 $-\mathrm{n}^{\circ} 63$.

Askenazy P., (2011), «Coût du travail dans l'industrie manufacturière en France et en Allemagne : des données peu fiables », miméo, février 2011.

Audric S., P. Givord et C. Prost, (2000), «Estimation de l'impact sur l'emploi non qualifié des mesures de baisse de charges », Revue économique, vol. 51, n 3, p. 513-522.

Barlet, M., D. Blanchet et T. Le Barbanchon, (2010), « Microsimulation et modèles d'agents : une approche alternative pour l'évaluation des politiques de l'emploi », Économie et Statistique, n429430, pp 51-76.

Bauduin N., L'Horty et F. Legendre, (2010), «Les baisses de cotisations sociales ultramarines », Revue Française d'Economie, n³, vol XXIV, janvier, pp 167-191.

Bauduin N., L'Horty et F. Legendre, (2011), «Réformer les baisses de cotisations sociales ultramarines », Travail et Emploi, $\mathrm{n}^{\circ} 125$, janvier-mars 2011.

Bunel M. et Y. L'Horty, (2011-a), «Pourquoi est-il si difficile d'évaluer les politiques publiques ?», Reflets et perspectives de la vie économique, 2011/1-2 (Tome L), pp 23-31.

Bunel M. et Y. L'Horty, (2011-b), Les effets des aides publiques aux Hôtels Cafés Restaurants et leurs interactions : une évaluation sur micro-données d'entreprises, Rapport pour le compte de la DARES, miméo.

Bunel M. et Y. L'Horty, (2012), «The effects of Social Security Payroll Tax Reductions on Employment : An Evaluation of the 2003 French Reform », Fiscal Studies, Forthcoming.

Bunel M., F. Gilles. et Y. L'Horty, (2010), « Les effets des allègements de cotisations sociales sur l'emploi et les salaires : une évaluation de la réforme de 2003 », Économie et Statistique n429-430, pp. 77-105.

Bur Y., (2008), « Mission d'information commune sur les exonérations de cotisations sociales », Rapport d'information de l'Assemblée Nationale, \# 1001.

Cahuc P., (2003), «Baisser les charges sociales, jusqu'où et comment ? », Revue Française d'Économie, vol. 17(3), pages 3-54.

Cahuc P., G. Cette et A. Zylberberg, (2008), Salaire minimum et bas revenus : comment concilier justice sociale et efficacité économique ? Rapport du Conseil d'Analyse Economique,

Chéron A., F. Langot et J-O. Hairault, (2005), « La baisse des charges en France. Un bon compromis entre emploi et productivité », Revue française d'économie. Volume 19 n4, 2005. pp. 3-40. 
Chéron A., F. Langot et J-O. Hairault, (2008), « A quantitative evaluation of payroll tax subsidies for low-wage workers: an equilibrium search approach », Journal of Public Economics, 92 (3-4).

Conseil d'Orientation pour l'Emploi, (2006), «Rapport au premier ministre relatif aux aides publiques», février 2006.

Conseil Supérieur de l'Emploi, des Revenus et de la Cohésion sociale, (1996), L'allègement des charges sociales sur les bas salaires, Collection des rapports au Premier ministre, La documentation Française.

Conseil Supérieur de l'Emploi, des Revenus et de la Cohésion sociale, (1999), Le SMIC, salaire minimum de croissance, La documentation Française.

Crépon B. et R. Desplatz, (2001), «Une nouvelle évaluation des effets des allègements de charges sociales sur les bas salaires », Économie et Statistique, n 348-08, p3-24.

Crépon B. et R. Desplatz, (2002), «Réduction des charges et emploi : évaluer la critique », Revue de l'OFCE, n ${ }^{\circ} 82$, juillet, p. 231-245.

DARES-DGTPE, (2005), « Les allègements de cotisations sociales patronales sur les bas salaires en France de 1993 à $2005 »$, miméo.

Demailly D., D. Marlat et L. Rioux, (2012), «Les déterminants du coût du travail en France », Insee Première, $\mathrm{n}^{\circ} 1393$.

Doisy S., Duchêne S. et C. Gianella, (2004), «Un modèle d'appariement avec hétérogénéité du facteur travail : un nouvel outil d'évaluation des politiques économiques », Économie et Prévision, n¹62, p. $1-22$.

Gafsi I., Y. L’Horty et F. Mihoubi, (2004), «Allègement du coût du travail et emploi peu qualifié : une réévaluation », in Méda D., Vennat F. (dir), Le travail non qualifié, permanences et paradoxes, collection Recherches, La Découverte.

GafsiI., Y. L’Horty et F. Mihoubi, (2005), « Réformer les exonérations de cotisations sociales sur les bas salaires », Revue française d'économie, 19(3), pp. 91-116.

Gianella P., (1999), «Elasticité de substitution entre qualifiés et non-qualifiés une comparaison des études économétriques partir des enquêtes DADS et ACEMO». INSEE DESE Document de travail 9912 bis

Goarant C. et L. Muller, (2011), «Les effets des hausses du Smic sur les salaires mensuels dans les entreprises de 10 salariés ou plus de 2006 à 2009 », Insee, Emploi et Salaire.

Hamermesh D., (1979), « New Estimates of the Incidence of the Payroll Tax », Southern Economic Journal, 45, p.1208-19.

Hamermesh D., (1993), Labor Demand, Princeton University Press.

Jacquot A, (1998), «Une maquette équilibre général pour analyse de la fiscalité du travail ronéotypé ». DARES 26 mai

Jamet S., (2005), «Allègements généraux de cotisations sociales et emploi peu qualifiés : de l'impact sectoriel à l'effet macro-économique », Revue Française d'Économie, vol. 19, n³, p. 57-90.

L'Horty Y., (2000), «Quand les hausses du Smic réduisent le coût du travail », Revue économique, 51(3).

L'Horty Y. (2001), « Baisse des cotisations sociales sur les bas salaires : une réévaluation », Économie et Statistique, 348, p. 25-31.

L'Horty (2006). «Dix ans d'évaluation des exonérations sur les bas salaires ». Connaissance de l'Emploi, n²4, janvier 2006.

Laffargue J.-P. et A. Saint-Martin, (1999), «Inégalités biais de progrès technique et imperfections de marché en France de 1974 à 1993 ». Economie et Prévision, n¹38-139, pp 89-109. 
Laffargue J-P., (1996), "Fiscalité, charges sociales, qualifications et emplois», Économie et Prévision, $\mathrm{n}^{\circ} 125-4$.

Laffargue J-P., (2000), «Effets et financement d'une réduction des charges sur les bas salaires », Revue économique, 51(3), p. 489-498.

Langot F., (2011), «Evaluer les politiques d'emploi : un plaidoyer pour une approche structurelle », Revue française d'économie, 24(1).

Laroque G. et B. Salanié, (2000), «Une décomposition du non-emploi en France», Économie et Statistique, $\mathrm{n}^{\circ} 331$.

Lehmann E., Marical F. et L. Rioux, (2011), « Labor Earnings Respond Differently to Income-Tax and Payroll-Tax Reforms », IZA Discussion Papers 6108, Institute for the Study of Labor (IZA).

Lhommeau B. et V. Rémy, (2010), « Les politiques d'allègements ont-elles un effet sur salariale des travailleurs à bas salaire? », Économie et Statistique, 429-430, p. 21-49.

Malinvaud E., (1998), Les cotisations sociales à la charge des employeurs : analyse économique, Rapport du CAE, La documentation Française.

Malinvaud E., (2002), «Sur l'agrégation des demandes de travail peu qualifiées ». Annales d'Economie et de Statistiques, ${ }^{\circ} 66$.

Ourliac B. et C. Nouveau, (2012), «Les allègements de cotisations sociales patronales sur les bas salaires en France de 1993 à 2009 », document d'étude de la DARES, n¹69, février.

Rapport du groupe d'experts Salaire Minimum Interprofessionnel de Croissance, décembre 2011.

Rapport du groupe d'experts Salaire Minimum Interprofessionnel de Croissance, décembre 2010.

Rapport du groupe d'experts Salaire Minimum Interprofessionnel de Croissance, décembre 2009.

Salanié B., (2000), «Une maquette analytique du marché du travail à long terme », Economie et Prévision, 146, pp1-13.

Seguin S., (2006b), «Les salariés au Smic en 2002 : un sur deux travaille dans une petite entreprise, un sur quatre gagne plus de 1,3 Smic horaire grâce à des compléments de salaire », Premières Synthèses, $\mathrm{n}^{\circ} 27.2$. 
Annexe 1.

\section{Les éléments inclus et exclus de l'assiette de vérification du Smic}

Tableau 1 : Assiette de vérification du SMIC

\begin{tabular}{|c|c|}
\hline ELEMENTS INCLUS & ELEMENTS EXCLUS \\
\hline $\begin{array}{l}\text { - Salaire de base } \\
\text { - Avantages en nature. } \\
\text { - Compensation pour réduction } \\
\text { d'horaire. } \\
\text { - Majorations diverses ayant le } \\
\text { caractére de fait d'un complément de } \\
\text { salaire (primes, indemnités, } \\
\text { remboursements de frais ne } \\
\text { correspondant pas à une dépense } \\
\text { effective...). } \\
\text { - Pourboires, gueltes... } \\
\text { - Primes de rendement individuelles } \\
\text { ou collectives (rendement global } \\
\text { d'une équipe). primes de production } \\
\text { ou de productivité constituant un } \\
\text { élément prévisible de rémunération. } \\
\text { - Primes de fin d'année pour le mois } \\
\text { où elles sont versées. } \\
\text { - Primes de vacances pour le mois où } \\
\text { elles sont versées. } \\
\text { - Primes de polyvalence. }\end{array}$ & $\begin{array}{l}\text { - Remboursements de frais } \\
\text { effectivement supportés. } \\
\text { - Primes forfaitaires destinées à } \\
\text { compenser les fras exposés par les } \\
\text { salaries du fait de leur prestation de } \\
\text { travail (primes de panier, d'outiliage, de } \\
\text { salissure, indemnités de petit ou grand } \\
\text { déplacement...). } \\
\text { - Majorations pour heures } \\
\text { supplémentaires. } \\
\text { - Majorations pour travail du dimanche, } \\
\text { des jours fériés et de nut. } \\
\text { - Primes d'ancienneté. } \\
\text { - Primes d'assiduité. } \\
\text { - Primes liées à la situation } \\
\text { géographique } \\
\text { (insularité, barrages, chantiers). } \\
\text { - Primes liées à des conditions } \\
\text { particulières de travaả (danger, froid, } \\
\text { insalubrité...) } \\
\text { - Primes collectives liées à la } \\
\text { production } \\
\text { globale de rentreprise, sa productivité } \\
\text { ou ses résultats. } \\
\text { - Primes de transport. } \\
\text { - Participation, intéressement. }\end{array}$ \\
\hline
\end{tabular}

Source : Seguin, 2006

Répartition des salariés "au SMIC" selon leur niveau de rémunération horaire effective

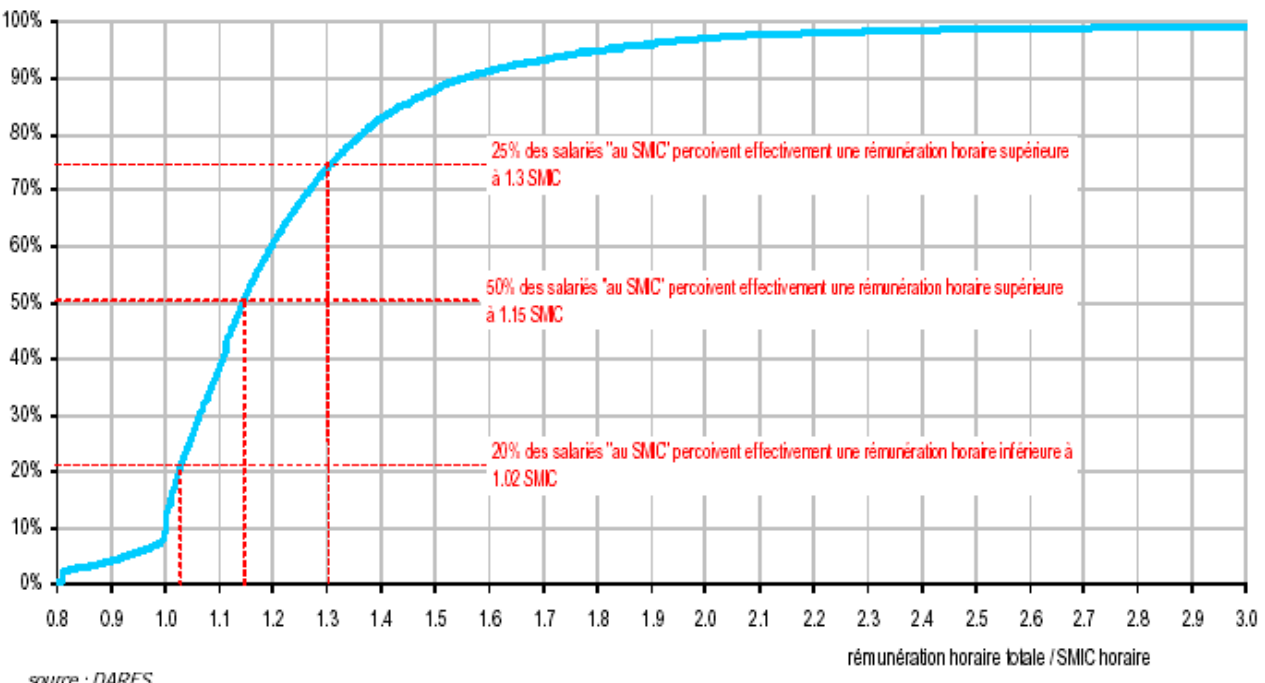

Source : Seguin, 2006 
3. Répartition des salariés dans les tranches salariales en multiples du Smic

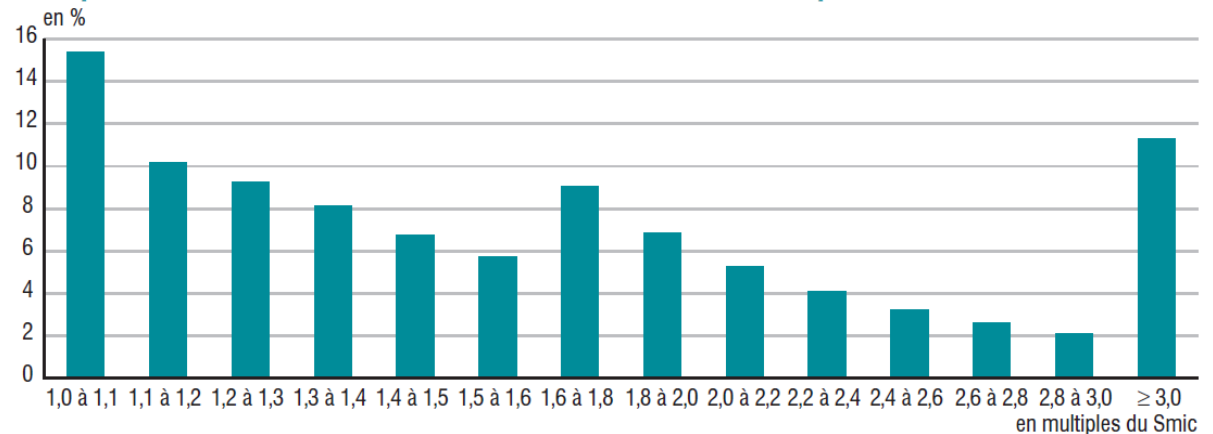
Champ : France métropolitaine, ensemble des salariés des entreprises de 10 salariés ou plus, sauf stagiaires, intérimaires; ensemble des secteurs sauf agriculture, administration, syndicats de copropriété, associations de loi 1901 de l'action sociale, activités des ménages, activités extraterritoriales, sauf entreprises du secteur de l'éducation, de la santé et de l'action sociale.

Source : Dares, enquêtes Acemo trimestrielles, calculs des auteurs.

Source : Goarant et Muller, 2011 
Annexe 2.

Tableau A1 : Répartition sectorielle des emplois à bas salaire

\begin{tabular}{|c|c|c|c|}
\hline NES36 & $\% 1,3$ Smic & $\% 1,6$ Smic & $\begin{array}{c}\text { \% de l'emploi } \\
\text { appartenant } \\
\text { aux secteurs } \\
\text { intenses en } \\
\text { main-d'œuvre }\end{array}$ \\
\hline Agriculture, sylviculture, pêche & $55,3 \%$ & $74,3 \%$ & $0,0 \%$ \\
\hline Industries agricoles et alimentaires & $42,7 \%$ & $67,1 \%$ & $0,0 \%$ \\
\hline Habillement, cuir & $57,9 \%$ & $74,6 \%$ & $99,4 \%$ \\
\hline Edition, imprimerie, reproduction & $22,9 \%$ & $41,8 \%$ & $0,0 \%$ \\
\hline Pharmacie, parfumerie et entretien & $12,1 \%$ & $24,9 \%$ & $0,0 \%$ \\
\hline Industries des équipements du foyer & $38,0 \%$ & $63,6 \%$ & $73,6 \%$ \\
\hline Industrie automobile & $14,3 \%$ & $34,4 \%$ & $0,0 \%$ \\
\hline Construction navale, aéronautique et ferroviaire & $11,1 \%$ & $24,8 \%$ & $0,0 \%$ \\
\hline Industries des équipements mécaniques & $23,1 \%$ & $47,5 \%$ & $0,0 \%$ \\
\hline Industries des équipements électriques et électroniques & $17,1 \%$ & $33,0 \%$ & $0,0 \%$ \\
\hline Industries des produits minéraux & $28,4 \%$ & $54,5 \%$ & $0,0 \%$ \\
\hline Industrie textile & $46,8 \%$ & $68,9 \%$ & $92,6 \%$ \\
\hline Industries du bois et du papier & $34,9 \%$ & $58,7 \%$ & $61,2 \%$ \\
\hline Chimie, caoutchouc, plastiques & $23,1 \%$ & $45,4 \%$ & $0,0 \%$ \\
\hline Métallurgie et transformation des métaux & $26,9 \%$ & $53,3 \%$ & $6,5 \%$ \\
\hline Industries des composants électriques et électroniques & $23,0 \%$ & $43,2 \%$ & $0,0 \%$ \\
\hline Production de combustibles et de carburants & $2,5 \%$ & $6,3 \%$ & $0,0 \%$ \\
\hline Eau, gaz, électricité & $4,7 \%$ & $13,9 \%$ & $0,0 \%$ \\
\hline Construction & $41,3 \%$ & $64,3 \%$ & $63,8 \%$ \\
\hline Commerce de gros & $8,8 \%$ & $50,3 \%$ & $0,0 \%$ \\
\hline Commerce de détail, réparations & $58,9 \%$ & $78,1 \%$ & $98,2 \%$ \\
\hline Transports & $32,3 \%$ & $52,2 \%$ & $6,3 \%$ \\
\hline Activités financières & $9,7 \%$ & $22,3 \%$ & $0,0 \%$ \\
\hline Activités immobilières & $34,4 \%$ & $58,3 \%$ & $0,0 \%$ \\
\hline Postes et télécommunications & $13,0 \%$ & $28,1 \%$ & $61,5 \%$ \\
\hline Conseils et assistance & $18,0 \%$ & $32,8 \%$ & $0,0 \%$ \\
\hline Services opérationnels & $57,0 \%$ & $75,0 \%$ & $78,3 \%$ \\
\hline Recherche et développement & $6,3 \%$ & $15,5 \%$ & $0,0 \%$ \\
\hline Hôtels et restaurants & $60,2 \%$ & $80,6 \%$ & $100,0 \%$ \\
\hline Activités récréatives, culturelles et sportives & $28,4 \%$ & $44,9 \%$ & $0,0 \%$ \\
\hline Services personnels & $67,9 \%$ & $85,4 \%$ & $99,7 \%$ \\
\hline Education & $31,8 \%$ & $51,0 \%$ & $0,0 \%$ \\
\hline Santé, action sociale & $27,5 \%$ & $53,0 \%$ & $0,0 \%$ \\
\hline
\end{tabular}

Source : Déclarations annuelles de données sociales, 2008, calculs des auteurs 
Annexe 3.

\section{Simulation sur des cas d'entreprise}

Une réforme des exonérations de cotisations sociales produit des effets différents selon les secteurs d'activité et selon les entreprises. Pour bien illustrer ces différences, nous avons également mené à bien des simulations au niveau individuel des entreprises avec la maquette SISMICs.

Le point de départ a été de réaliser une petite enquête auprès de quelques entreprises des secteurs intenses en main-d'œuvre ${ }^{15}$. Cette enquête portait sur la distribution des rémunérations, sur celle du temps de travail par tranche de salaire, et sur des informations générales sur l'entreprise. Dix entreprises ont répondu à l'enquête ce qui n'est pas du tout représentatif mais néanmoins suffisant pour notre propos qui est d'illustrer les différences entre les entreprises dans les effets d'une réforme. Ces entreprises ont des effectifs compris entre 57 et 79000 salariés et relèvent de domaine d'activité variés (propreté, travail temporaire, céramiques, manutention de bagages). Puisque leur distribution des salaires sont différentes, elles sont plus ou moins concernées par les exonérations de cotisations sociales. Dans quatre entreprises, plus de $95 \%$ des salariés sont rémunérés en deçà de 1,6 Smic tandis que dans une autre, seulement $26 \%$ des salariés sont concernés par les exonérations générales de cotisations sociales. Les montants d'exonération par salarié exonéré et par an sont également variés. Ils vont de $1137 €$ à $3886 €$ selon les entreprises. Ces exonérations permettent de diminuer le coût du travail d'un pourcentage qui est compris entre 1,5\% et $18,4 \%$. Le budget des exonération est donc lui aussi variable. Il va de $200000 €$ à plus de 110 millions d' $€$ selon les entreprises. Le taux d'exonération moyen varie de $7 \%$ à $25 \%$. \%. Ces études de cas soulignent un élément important : un dispositif unique d'exonération produit des effets différents selon les entreprises y compris au sein d'un même secteur d'activité.

Sur cette base, nous avons évalué les effets sur l'emploi du dispositif d'exonération de cotisations sociales en simulant sa suppression pure et simple et en supposant que chaque entreprise réagirait de la même façon. Cette réaction uniforme est donnée par l'élasticité de la demande de travail à son coût que nous avons supposé égale à la valeur que nous avons estimée en moyenne sur l'ensemble des entreprises dans notre étude précédente (Bunel et L'Horty [2012]. L'élasticité est donc la même pour toutes les entreprises et égale à 0,516. En étant confrontées au même choc, la suppression des exonérations générales, et en réagissant par hypothèse de la même façon, les entreprises de notre enquête voient leur emploi reculer de $0,8 \%$ à $8,1 \%$, c'est-à-dire un facteur allant de 1 à 10 !

\footnotetext{
${ }^{15}$ Nous remercions les responsables des entreprises ayant bien voulu répondre à cette enquête.
} 
Tableau A2. Simulation d'une suppression des exonérations générales sur des cas d'entreprises

\begin{tabular}{|c|c|c|c|c|c|c|c|c|c|c|}
\hline Domaine d'activité & $\begin{array}{c}\text { A } \\
\text { Travail } \\
\text { temporaire }\end{array}$ & $\begin{array}{c}\text { B } \\
\text { Propreté }\end{array}$ & $\begin{array}{c}\text { C } \\
\text { Propreté }\end{array}$ & $\begin{array}{c}\text { D } \\
\text { Communication }\end{array}$ & $\begin{array}{c}\mathbf{E} \\
\text { Travail } \\
\text { temporaire }\end{array}$ & $\begin{array}{c}\text { F } \\
\text { Propreté }\end{array}$ & $\begin{array}{c}\text { G } \\
\text { Céramiques }\end{array}$ & $\begin{array}{c}\text { H } \\
\text { Vaisselle }\end{array}$ & $\begin{array}{c}\text { I } \\
\text { Manutention } \\
\text { bagages }\end{array}$ & $\begin{array}{c}\text { J } \\
\text { Propreté }\end{array}$ \\
\hline $\begin{array}{l}\text { Nombre de salariés dans } \\
\text { l'entreprise }\end{array}$ & 78676 & 68795 & 35197 & 23451 & 7183 & 1223 & 164 & 135 & 76 & 57 \\
\hline $\begin{array}{l}\text { Nombre de salariés } \\
\text { concernés par les } \\
\text { exonérations }\end{array}$ & 54209 & 66349,26 & 34057 & 21865,25 & 3735 & 321 & 151 & 107 & 74 & 55,86 \\
\hline $\begin{array}{l}\text { Part des salariés concernés } \\
\text { par les exonérations }\end{array}$ & $69 \%$ & $96 \%$ & $97 \%$ & $93 \%$ & $52 \%$ & $26 \%$ & $92 \%$ & $79 \%$ & $97 \%$ & $98 \%$ \\
\hline Exonération par tête & 1941,94 & 1727,64 & 3095,54 & 3886,58 & 1137,16 & 1258,69 & 2043,12 & 3368,82 & 3282,04 & 2753,27 \\
\hline $\begin{array}{l}\text { Budget exonérations (en } \\
\text { millions) }\end{array}$ & 105,27 & 114,62 & 105,42 & 85 & 4,25 & 0,4 & 0,31 & 0,36 & 0,24 & 0,2 \\
\hline $\begin{array}{l}\text { Variation en \% du coût du } \\
\text { travail }\end{array}$ & $4,90 \%$ & $13,59 \%$ & $15,90 \%$ & $18,36 \%$ & $3,80 \%$ & $1,50 \%$ & $7,00 \%$ & $11,30 \%$ & $12,80 \%$ & $15,45 \%$ \\
\hline $\begin{array}{l}\text { Salaire mensuel moyen des } \\
\text { exonérés }\end{array}$ & $1860,70 €$ & $821,10 €$ & $1270,20 €$ & $1274 €$ & $1126,50 €$ & $1491,10 €$ & $1783,80 €$ & $1589,50 €$ & $1606,20 €$ & $1184,90 €$ \\
\hline Taux d'exonération apparent & $8,70 \%$ & $17,53 \%$ & $20,30 \%$ & $25,42 \%$ & $8,40 \%$ & $7,00 \%$ & $9,50 \%$ & $17,70 \%$ & $17,00 \%$ & $19,36 \%$ \\
\hline Pertes d'emplois en $\%$ & $3 \%$ & $6 \%$ & $8 \%$ & $8 \%$ & $2 \%$ & $1 \%$ & $4 \%$ & $6 \%$ & $7 \%$ & $8 \%$ \\
\hline $\begin{array}{l}\text { Economie budgétaire par } \\
\text { emploi détruit (en milliers } \\
d^{\prime} € \text { ) }\end{array}$ & 53,33 & 27,25 & 36,87 & 43,85 & 30,78 & 42,18 & 52,37 & 46,37 & 49,11 & 34,24 \\
\hline
\end{tabular}

Source : Enquête auprès d'entreprises de main-d'œuvre, et maquette SISMICs 
12-1. Etre meilleur Apprenti de France : Quels effets sur l'accès à l'emploi ? Les enseignements de deux expériences contrôlées sur des jeunes d'Ile-de-France Pascale Petit, Florent Fremigacci, Loïc du Parquet, Guillaume Pierne

12-2. L'intermédiation financière dans l'analyse macroéconomique : Le défi de la crise Eleni Iliopoulos, Thepthida Sopraseuth

12-3. Evaluer un dispositif sectoriel d'aide à l'emploi : L'exemple des hôtels cafés restaurants de 2004 à 2009

Mathieu Bunel 
11-1. Les effets du lieu de résidence sur l'accès à l'emploi: Une expérience contrôlée sur des jeunes qualifiés en Ile-de-France

Yannick L'Horty, Emmanuel Duguet, Loïc du Parquet, Pascale Petit, Florent Sari

11-2. Comment développer les emplois favorables à la biodiversité en Ile-De-France ? Jean de Beir, Céline Emond, Yannick L'Horty, Laëtitia Tuffery

11-3. Être mobile pour trouver un emploi ?Les enseignements d'une expérimentation en région parisienne

Loïc du Parquet, Emmanuel Duguet, Yannick L’Horty, Pascale Petit, Florent Sari

11-4. Ce que font les villes pour les ménages pauvres. Résultats d'une enquête nationale sur les communes de plus de 20000 habitants

Denis Anne, Céline Emond, Yannick L’Horty

11-5. Discriminations à l'embauche des jeunes franciliens et intersectionalité du sexe et de l'origine: Les résultats d'un testing

Pascale PETIT, Emmanuel DUGUET, Yannick L'HORTY, Loïc du PARQUET, Florent

SARI

11-6. Les effets du bénévolat sur l'accès à l'emploi. Une expérience contrôlée sur des jeunes qualifiés d'Ile-de-France

Jonathan Bougard, Thomas Brodaty, Céline Emond, Yannick L’Horty, Loïc du Parquet et

Pascale Petit

11-7. « 10000 permis pour réussir ». Evaluation quantitative

Yannick L’Horty, Emmanuel Duguet, Sophie Kaltenmark, Pascale Petit

11-8. Why is there a faster return to work near the border?

Jonathan Bougard

11-9. Evaluer l'impact d'un micro-programme social : une étude de cas expérimentale Yannick L'Horty, Emmanuel Duguet, Pascale Petit

11-10. Les effets des aides publiques aux Hôtels Cafés Restaurants et leurs interactions : Une évaluation sur micro-données d'entreprises

Mathieu Bunel, Yannick L'Horty

11-11. Pourquoi tant de chômeurs à Paris

Yannick L'Horty, Florent Sari

11-12. LE WIKI IO : Réduire les risques de décrochage et d'abandon à la sortie du collège

Solène Coursaget, Emmanuel Duguet, Yannick L'Horty, Pascale Petit, Emmanuel Quenson

11-13. Le grand Paris de l'emploi

Yannick L'Horty, Florent Sari

11-14. Quelle politique publique pour protéger la biodiversité Jean De Beir, Céline Emond, Yannick L'Horty, Laetitia Tuffery 
La Fédération de recherche CNRS Travail, Emploi et Politiques Publiques (TEPP, FR $\mathbf{n}^{\circ} \mathbf{3 1 2 6}$ ) réunit des centres de recherche en économie et sociologie :

- Le Centre d'Etudes des Politiques Economiques de l'université d'Evry, EPEE, Université d'Evry Val d'Essonne

- Le Centre Pierre Naville, CPN, Université d'Evry Val d'Essonne

- Le Centre de Recherche en Economie et Management, CREM, Université de Caen Basse Normandie et Université de Rennes 1

- L'Equipe de Recherche sur les Marchés, l'Emploi et la Simulation, ERMES, Université deParis II Panthéon-Assas

- L'Equipe de Recherche sur l'Utilisation des Données Temporelles en Economie, ERUDITE, Université de Paris-Est Créteil et Université de Paris-Est Marne-la-Vallée

- Le Groupe d'Analyse des Itinéraires et des Niveaux Salariaux, GAINS, Université du Maine

La Fédération TEPP rassemble 150 chercheurs et enseignants-chercheurs, 140 doctorants et 40 chercheurs associés, qui étudient les mutations du travail et de l'emploi en relation avec les choix des entreprises et analysent les politiques publiques en mobilisant les nouvelles méthodes d'évaluation. 